\title{
Highly directive current distributions: General theory
}

\section{Citation}

Margetis, Dionisios, George Fikioris, John M. Myers, and Tai Tsun Wu. 1998. "Highly

Directive Current Distributions: General Theory." Physical Review E 58 (2): 2531-47. https:// doi.org/10.1103/physreve.58.2531.

\section{Permanent link}

http://nrs.harvard.edu/urn-3:HUL.InstRepos:41555808

\section{Terms of Use}

This article was downloaded from Harvard University's DASH repository, and is made available under the terms and conditions applicable to Other Posted Material, as set forth at http:// nrs.harvard.edu/urn-3:HUL.InstRepos:dash.current.terms-of-use\#LAA

\section{Share Your Story}

The Harvard community has made this article openly available.

Please share how this access benefits you. Submit a story.

Accessibility 


\title{
Highly directive current distributions: General theory
}

\author{
Dionisios Margetis, ${ }^{1}$ George Fikioris, ${ }^{2}$ John M. Myers, ${ }^{1}$ and Tai Tsun $\mathrm{Wu}^{1}$ \\ ${ }^{1}$ Gordon McKay Laboratory, Harvard University, Cambridge, Massachusetts 02138-2901 \\ ${ }^{2}$ Air Force Research Laboratory, Sensors Directorate, Hanscom Air Force Base, Massachusetts 01731
}

(Received 19 November 1997)

\begin{abstract}
A theoretical scheme for studying the properties of localized, monochromatic, and highly directive classical current distributions in two and three dimensions is formulated and analyzed. For continuous current distributions, it is shown that maximizing the directivity $D$ in the far field while constraining $C=N / T$, where $N$ is the integral of the square of the magnitude of the current density and $T$ is proportional to the total radiated power, leads to a Fredholm integral equation of the second kind for the optimum current. This equation is a useful analytical tool for studying currents that produce optimum directivities above the directivity of a uniform distribution. Various consequences of the present formulation are examined analytically for essentially arbitrary geometries of the current-carrying region. In particular, certain properties of the optimum directivity are derived and differences between the continuous and discrete cases are pointed out. When $C \rightarrow \infty$, the directivity tends to infinity monotonically, in accord with Oseen's "Einstein needle radiation.",

[S1063-651X(98)02108-4]
\end{abstract}

PACS number(s): 41.20.Bt, 03.40.Kf

\section{INTRODUCTION}

An old question in classical electromagnetism was: For a current-carrying region of given shape and size and for a given operating frequency, what is the maximum directivity obtainable? This question was addressed as early as 1922 by Oseen [1]. Starting with Maxwell's equations, he essentially showed that an arbitrarily small current-carrying region can give rise to an arbitrarily narrow radiation pattern. Oseen termed this theoretical possibility "Einstein needle radiation,' referring to a 1909 paper by Einstein on the quantum nature of radiation [2]. Some later authors were apparently not aware of Oseen's fundamental, yet counterintuitive, result. According to Bloch, Medhurst, and Pool [3], it was forgotten and rediscovered twice after 1922. In 1943, for example, La Paz and Miller [4] claimed to have found a current distribution in a linear radiator that would give maximum directivity. Three years later, Bouwkamp and de Bruijn [5] demonstrated that La Paz and Miller had made an error: If one can choose the linear current distribution at will, there is no finite limit to the directivity of the radiator.

Also during the 1940s, several authors noticed that one must pay dearly for very large directivities. A specific second question is: When the directivity is very large, what is the price one pays? Perhaps the first author to address this question in a systematic manner was Chu [6]. Briefly, Chu introduced a quality factor $Q$, which he computed from the field distribution on a spherical surface enclosing an unspecified radiating system. By analogy with circuit theory, Chu related his $Q$ to bandwidth but noted that "It is understood that the physical interpretation of $Q$ as so computed becomes rather vague whenever the value of $Q$ is low." Through a study of his $Q$, Chu concluded that very large directivities lead to extremely small bandwidths and extremely low efficiencies, so that very large directivities, though possible in theory, are impractical.

Chu's ideas have been discussed, extended, and reexamined by many authors [7-9]. From the more recent works, one sees that a surrounding spherical surface remains a common device for addressing questions of the above type. Furthermore, variably defined $Q$ 's, frequently different from Chu's, still seem to be the most popular constraining quantities. Other authors, however, have severely criticized the relation between such $Q$ 's and bandwidth $[10,11]$. Investigations with commonly used $Q$ 's have pointed out anomalies or proposed remedies to such anomalies [10-13]. In Ref. [11], Rhodes called $Q$ “one of the most misused and misunderstood concepts in electrical engineering.",

It has been stressed (see, for example, Refs. [3,14,15]) that there is no precise threshold for the directivity that can be obtained in practice and that higher-than-conventional directivities can be obtained without running into severe difficulties. Indeed, recent investigations have focused on schemes for obtaining large directivities. These include the use of high-temperature superconductors [16] in conventional types of antennas or their feeding structures, as well as schemes $[15,17,18]$ that utilize the resonant properties of unconventional types of antennas.

The discussions above lead to a third, even more complicated question: How large a directivity can one obtain without paying dearly? Due to the unclear relation between $Q$ (defined in any of the usual ways) and the bandwidth when the value of the former is low, it is preferable to address this question by methods other than those originating from Chu. In reality, this rather vague question cannot have a simple answer, even from a strictly theoretical point of view. The primary reason is that current distributions cannot be chosen at will.

A fourth, more tractable question is: If one is free to choose the current distribution in a bounded region of given shape and size, how large a directivity can one obtain without paying dearly? Investigation of this question, a task that does not seem to have been addressed adequately, is a first step toward answering the preceding one and naturally calls for the formulation of a constrained maximization problem. 
The purpose of the present paper is to discuss a theoretical framework for examining the fourth question posed above and to study analytically the general consequences of this model. In the case of continuous current distributions, the constraining quantity $C$ is defined to be $N / T$, where $N$ is the integral of the square of the magnitude of the current density and $T$ is proportional to the total radiated power. It is noteworthy that, for a three-dimensional line source, this ratio becomes identical to Taylor's "supergain ratio" [19] when point sources of current are assumed to be isotropic radiators. The constraint $C$ has been used in various contexts by others [20-29], but their approaches or objectives differ from the present ones. The advantages of the employed constraint are readily recognized: It is a well-defined quantity, being directly obtainable from the currents instead of the fields on the surface of a surrounding sphere, and it is specific to the particular radiating system. Furthermore, it leads to a wellposed optimization problem. For most cases of practical interest, $C$ measures the efficiency of the radiating system and is therefore a natural constraint for the physical problem under investigation.

This paper is organized as follows. Section II proceeds from Maxwell's equations to state the optimization problem for both continuous and discrete current distributions. In Sec. III it is shown that the optimum continuous current can be conveniently identified as the solution to a linear integral equation of the Fredholm type. In Sec. IV some fundamental properties of the optimum directivity as a function of the constraint are derived as direct consequences of the formulation developed herein. In particular, it is shown that the integral equation yields known properties in certain limiting cases. Also contained in Sec. IV are properties that, though physically intuitive, have apparently not been quantified in previous analyses. For all reasonable purposes, the analytical properties discussed in this section are independent of the geometry of the current-carrying region. These considerations render the present approach distinctly different from rigorous analyses concerned with computational schemes for the numerical approximation of optimum solutions to radiation problems, such as the important work by Angell and Kleinman [25]. (See also Refs. [26,27].) Finally, Appendixes A-D provide the mathematical framework, along with the treatments of a few mathematically demanding subtleties and Appendix E describes a few cases where the aforementioned integral equation can be solved exactly.

It is emphasized that, due to the assumption that the current distribution can be chosen freely, the model studied in Secs. II-IV is idealized. For example, no attempt is made there to enforce any "realizability conditions," such as boundary conditions on three-dimensional current densities. This latter point is discussed in Sec. V. In practice, of course, one must specify means to maintain the desired currents. In general, this becomes increasingly difficult as the directivity grows and is beyond the scope of this paper. Nonetheless, a conjecture regarding the realization of optimum current distributions in closed loops is attempted in the discussion of Sec. V.

As also explained in Sec. V, the present analysis is intended as a tool for the study of currents that give rise to the Einstein needle radiation. The intricate nature of such currents, though perhaps not imminently practical from the en- gineering point of view, is physically attractive. This extreme case needs to be further understood.

\section{FORMULATION}

Let $\mathbf{J}(\mathbf{r}, t)=\operatorname{Re}\left\{\mathbf{J}(\mathbf{r}) e^{-i \omega t}\right\}$ denote a continuous threedimensional current distribution in a homogeneous and isotropic space. $\mathbf{J}(\mathbf{r})$ is assumed to be localized, i.e., to vanish identically outside of some bounded region $\mathcal{G}$, and to satisfy the condition

$$
N=V_{\mathcal{G}}^{-1} \int_{\mathcal{G}} d \mathbf{r}|\mathbf{J}(\mathbf{r})|^{2}<\infty,
$$

where $V_{\mathcal{G}}$ denotes the volume of region $\mathcal{G}\left(V_{\mathcal{G}}=\int_{\mathcal{G}} d \mathbf{r}\right)$ and is employed for normalization purposes: When the current has a unit magnitude everywhere in $\mathcal{G}, N$ is equal to 1 . In particular, when $\mathcal{G}$ is a curve $\mathbf{r}=\mathbf{r}(s)$ ( $s$ is the arc length), $V_{\mathcal{G}}$ denotes its length and Eq. (2.1) is understood as a condition on an integral in one dimension, with $d \mathbf{r} \equiv d s$. The quantity $\sqrt{N}$ will be referred to as "the current norm."

With the $e^{-i \omega t}$ time dependence suppressed, the ensuing electric and magnetic fields $\mathbf{E}(\mathbf{r})$ and $\mathbf{B}(\mathbf{r})$ outside $\mathcal{G}$ satisfy the homogeneous Maxwell equations

$$
\begin{gathered}
\boldsymbol{\nabla} \times \mathbf{E}(\mathbf{r})=i \omega \mathbf{B}(\mathbf{r}), \\
\boldsymbol{\nabla} \times \mathbf{B}(\mathbf{r})=-i \omega \epsilon \mu \mathbf{E}(\mathbf{r}),
\end{gathered}
$$

where $\epsilon$ and $\mu$ are the dielectric permittivity and magnetic permeability, respectively. With the introduction of the familiar vector and scalar potentials $\mathbf{A}$ and $\Phi$, the electromagnetic field can be expressed as

$$
\begin{gathered}
\mathbf{E}=i \omega \mathbf{B}-\boldsymbol{\nabla} \Phi, \\
\mathbf{B}=\boldsymbol{\nabla} \times \mathbf{A} .
\end{gathered}
$$

Calculations are carried out in the Lorentz gauge, where

$$
\boldsymbol{\nabla} \cdot \mathbf{A}=i \omega \mu \epsilon \Phi .
$$

Although any current polarization can be treated straightforwardly, it best serves the purposes of this analysis to consider a current $\mathbf{J}$ parallel to a fixed axis, say, $\hat{\mathbf{z}}$ :

$$
\mathbf{J}(\mathbf{r})= \begin{cases}J(\mathbf{r}) \hat{\mathbf{z}} & \text { inside } \mathcal{G} \\ 0 & \text { outside } \mathcal{G}\end{cases}
$$

This choice leads to

$$
\mathbf{A}(\mathbf{r})=\mu \int_{\mathcal{G}} d \mathbf{r}^{\prime} \frac{e^{i k\left|\mathbf{r}-\mathbf{r}^{\prime}\right|}}{4 \pi\left|\mathbf{r}-\mathbf{r}^{\prime}\right|} J\left(\mathbf{r}^{\prime}\right) \hat{\mathbf{z}}
$$

where $k=\omega / c$ is the wave number and $c=(\epsilon \mu)^{-1 / 2}$ is the velocity of light. The electric and magnetic fields are obtained via direct differentiation of $\mathbf{A}(\mathbf{r})$, as implied by Eqs. (2.5) and (2.3). Of interest is the field in the radiation zone, described by the leading terms of the asymptotic expansions of $\mathbf{E}(\mathbf{r})$ and $\mathbf{B}(\mathbf{r})$ when $k r=k|\mathbf{r}| \rightarrow \infty$. In the spherical coordinate system $(r, \theta, \phi)$ defined with respect to the $z$ axis, 


$$
\begin{aligned}
& \mathbf{E}(\mathbf{r}) \sim-i \omega \mu \frac{e^{i k r}}{4 \pi r} V_{\mathcal{G}} \psi(\hat{\mathbf{r}}) \sin \theta \hat{\boldsymbol{\theta}}, \\
& \mathbf{B}(\mathbf{r}) \sim-i \mu k \frac{e^{i k r}}{4 \pi r} V_{\mathcal{G}} \psi(\hat{\mathbf{r}}) \sin \theta \hat{\boldsymbol{\phi}},
\end{aligned}
$$

where

$$
\psi(\hat{\mathbf{r}})=\frac{1}{V_{\mathcal{G}}} \int_{\mathcal{G}} d \mathbf{r}^{\prime} J\left(\mathbf{r}^{\prime}\right) e^{-i k \hat{\mathbf{r}} \cdot \mathbf{r}^{\prime}}, \quad \hat{\mathbf{r}}=r^{-1} \mathbf{r} .
$$

Evaluation of the Poynting vector $\mathbf{S}=\frac{1}{2} \mathbf{E} \times \mathbf{H}^{*}$ (where the asterisk indicates complex conjugation) yields the power flux

$$
S_{r}(\hat{\mathbf{r}})=\frac{\omega^{2} \mu}{2 c} \frac{1}{(4 \pi r)^{2}} V_{\mathcal{G}}^{2}|\psi(\hat{\mathbf{r}})|^{2} \sin ^{2} \theta .
$$

The total radiated power is

$$
P_{T}=\frac{\omega^{2} \mu}{8 \pi c} V_{\mathcal{G}}^{2} T, \quad T=\frac{1}{4 \pi} \int_{(4 \pi)} d \Omega \sin ^{2} \theta|\psi(\hat{\mathbf{r}})|^{2},
$$

where $d \Omega=\sin \theta d \theta d \phi$ denotes the infinitesimal solid angle at $(\theta, \phi)$. Substituting $\psi(\hat{\mathbf{r}})$ from Eq. (2.11) into Eq. (2.13), interchanging the order of integration, and performing the $d \Omega$ integration result in

$$
T=\frac{1}{V_{\mathcal{G}}^{2}} \int_{\mathcal{G}} d \mathbf{r} \int_{\mathcal{G}} d \mathbf{r}^{\prime} J^{*}(\mathbf{r}) K\left(\mathbf{r}, \mathbf{r}^{\prime}\right) J\left(\mathbf{r}^{\prime}\right) .
$$

The associated three-dimensional kernel (derived in Appendix A) reads

$$
\begin{aligned}
K\left(\mathbf{r}, \mathbf{r}^{\prime}\right)= & K^{(3)}\left(\mathbf{r}, \mathbf{r}^{\prime}\right) \\
= & {\left[1-(\hat{\mathbf{z}} \cdot \hat{\mathbf{R}})^{2}\right] \frac{\sin (k R)}{k R} } \\
& -\left[1-3(\hat{\mathbf{z}} \cdot \hat{\mathbf{R}})^{2}\right]\left[\frac{\sin (k R)}{(k R)^{3}}-\frac{\cos (k R)}{(k R)^{2}}\right] \\
= & {\left[1-(\hat{\mathbf{z}} \cdot \hat{\mathbf{R}})^{2}\right] j_{0}(k R)-\left[1-3(\hat{\mathbf{z}} \cdot \hat{\mathbf{R}})^{2}\right] \frac{j_{1}(k R)}{k R}, }
\end{aligned}
$$

where

$$
\hat{\mathbf{R}}=|\mathbf{R}|^{-1} \mathbf{R}, \quad R=|\mathbf{R}|, \quad \mathbf{R}=\mathbf{r}-\mathbf{r}^{\prime},
$$

and $j_{0}, j_{1}$ denote the spherical Bessel functions of order 0 and $1 . K^{(3)}\left(\mathbf{r}, \mathbf{r}^{\prime}\right)$ is real and symmetric. When $\mathcal{G}$ lies in a plane perpendicular to $\hat{\mathbf{z}}, K^{(3)}\left(\mathbf{r}, \mathbf{r}^{\prime}\right)$ reduces to

$$
\begin{aligned}
K^{(3)}(k R) & =\frac{\sin (k R)}{k R}+\frac{\cos (k R)}{(k R)^{2}}-\frac{\sin (k R)}{(k R)^{3}} \\
& =j_{0}(k R)-\frac{j_{1}(k R)}{k R} .
\end{aligned}
$$

The two-dimensional case results from Eq. (2.8) with the assumption of a current distribution that is invariant in $z$. Accordingly, the potential associated with a current distribution $J(\mathbf{r})$ in a two-dimensional region $\mathcal{G}$ reads

$$
A(\mathbf{r})=\frac{i \mu}{4} \int_{\mathcal{G}} d \mathbf{r}^{\prime} H_{0}^{(1)}\left(k\left|\mathbf{r}-\mathbf{r}^{\prime}\right|\right) J\left(\mathbf{r}^{\prime}\right) .
$$

The corresponding scalar field in the radiation zone is [30]

$$
E(\mathbf{r}) \sim-\omega \mu V_{\mathcal{G}} \frac{e^{i(k r-\pi / 4)}}{\sqrt{8 \pi k r}} \psi(\hat{\mathbf{r}})
$$

where $\mathbf{r}=(x, y)$ is the two-dimensional position vector, $\hat{\mathbf{r}}$ $=(\cos \phi, \sin \phi)$, and $\psi(\hat{\mathbf{r}})$ is given by an equation of the form (2.11). The power flux $S_{r}(\hat{\mathbf{r}})$ and the total radiated power $P_{T}$ are given by

$$
\begin{gathered}
S_{r}(\hat{\mathbf{r}})=\frac{\omega \mu}{16 \pi r} V_{\mathcal{G}}^{2}|\psi(\hat{\mathbf{r}})|^{2}, \\
P_{T}=\frac{\omega \mu}{8} V_{\mathcal{G}}^{2} T, \quad T=\frac{1}{2 \pi} \int_{0}^{2 \pi} d \phi|\psi(\hat{\mathbf{r}})|^{2} .
\end{gathered}
$$

$T$ can be recast in the form (2.14) with the real and symmetric kernel

$$
K\left(\mathbf{r}, \mathbf{r}^{\prime}\right)=K^{(2)}\left(\mathbf{r}, \mathbf{r}^{\prime}\right)=K^{(2)}(k R)=J_{0}(k R),
$$

where $J_{0}$ is the Bessel function of zeroth order.

It is not difficult to modify the foregoing analysis in order to treat discrete distributions consisting of $M$ electric dipoles or current filaments of amplitudes $\left\{I_{j}\right\}$ at fixed positions $\left\{\mathbf{r}_{j}\right\}$ $(j=1, \ldots, M)$ in two or three dimensions, respectively. The square of the current norm is then defined as

$$
N=M^{-1} \sum_{j=1}^{M}\left|I_{j}\right|^{2}<\infty .
$$

With $\psi(\hat{\mathbf{r}})$ in Eq. (2.11) being simply replaced by

$$
\psi(\hat{\mathbf{r}})=M^{-1} \sum_{j=1}^{M} I_{j} e^{-i k \hat{\mathbf{r}} \cdot \mathbf{r}_{j}}
$$

all of the preceding expressions for the far field, the power flux, and the total radiated power in terms of $\psi(\hat{\mathbf{r}})$ remain valid. The discrete analog of Eq. (2.14) reads

$$
T=M^{-2} \sum_{j=1}^{M} \sum_{l=1}^{M} I_{j}^{*} K\left(\mathbf{r}_{j}, \mathbf{r}_{l}\right) I_{l},
$$

where $K$ is equal to $K^{(3)}$ or $K^{(2)}$. This equation shows that the continuous kernel $K\left(\mathbf{r}, \mathbf{r}^{\prime}\right)$ represents the interference (cross) term in the total power radiated by two parallel current elements (dipoles or filaments) of unit amplitudes, located at positions $\mathbf{r}$ and $\mathbf{r}^{\prime}\left(\mathbf{r} \neq \mathbf{r}^{\prime}\right)$.

Finally, let $\hat{\mathbf{r}}_{0}$ be a direction of preference in the two- or three-dimensional space. For continuous or discrete current distributions, define the quantities 


$$
\begin{gathered}
D=P / T, \quad P=\left|\psi\left(\hat{\mathbf{r}}_{0}\right)\right|^{2}, \\
C=N / T .
\end{gathered}
$$

With an additional factor of $\left[1-\left(\hat{\mathbf{r}}_{0} \cdot \hat{\mathbf{z}}\right)^{2}\right]$ in three dimensions, $D$ is the directivity in $\hat{\mathbf{r}}_{0}$. For continuous surface currents flowing in highly conducting materials or currents in dipoles, $C$ measures the efficiency of the radiating system because $N$ is proportional to the Ohmic losses.

The optimization problem can now be stated as follows.

Problem II.1. Given a current-carrying region $\mathcal{G}$ (or $\left\{\mathbf{r}_{j}\right\}, j=1, \ldots, M$, in the case of discrete current distributions) and $\hat{\mathbf{r}}_{0}\left(\hat{\mathbf{r}}_{0} \neq \hat{\mathbf{z}}\right.$ in three dimensions $)$, determine admissible continuous current distributions $J(\mathbf{r})=J_{\text {opt }}(\mathbf{r})$ (or discrete $\left\{I_{j}=I_{j}^{(\text {opt })}\right\}$ ) that maximize $\hat{D}[J]$ (or $\hat{D}[\mathbf{I}]$ ) for fixed $\hat{C}[J]=C($ or $\hat{C}[\mathbf{I}]=C)$.

The caret over a capital letter means a functional in $J$ or a function of $\mathbf{I}=\left[I_{j}\right]$. "Admissible" distributions are continuous (or discrete) localized currents whose norm exists and is finite and nonzero. Because multiplication of the current distribution by a constant leaves $\hat{D}[J]$ and $\hat{C}[J]$ unaltered, this problem does not yield a unique optimum current. For the appropriate values of $C$, any such optimum current can be viewed as the unique solution to a corresponding problem.

Problem II.2. Given a current-carrying region $\mathcal{G}$ (or $\left.\left\{\mathbf{r}_{j}\right\}, \quad j=1, \ldots, M\right)$ and $\hat{\mathbf{r}}_{0}\left(\hat{\mathbf{r}}_{0} \neq \hat{\mathbf{z}}\right.$ in three dimensions $)$, determine the admissible continuous current distribution $J_{\text {opt }}(\mathbf{r})$ (or discrete $\left\{I_{j}^{(\mathrm{opt})}\right\}$ ) that maximizes the power-flux functional $\hat{P}[J]$ (or $\hat{P}[\mathbf{I}]$ ) for fixed $\hat{N}[J]=N($ or $\hat{N}[\mathbf{I}]=N)$, $\hat{T}[J]=T($ or $\hat{T}[\mathbf{I}]=T)$, and phase $\operatorname{Arg} \psi\left(\hat{\mathbf{r}}_{0}\right)$.

In the case of discrete current distributions, the constraint has an upper (finite) bound, as also discussed by Lo et al. [24], who use $C / M$ as a constraint. This upper bound becomes infinite for a continuous current distribution $[1,5]$. In Appendixes B and $\mathrm{C}$ both of these assertions are shown to be direct consequences of solving Problem II.1. In order to simplify the analysis, Problem II.2 is addressed first.

\section{EQUATION FOR THE OPTIMUM CURRENT}

\section{A. Continuous case}

For mathematical convenience, an overall phase is chosen for the continuous current distribution $J(\mathbf{r})$ so that $\operatorname{Arg} \psi\left(\hat{\mathbf{r}}_{0}\right)=0$, permitting

$$
\sqrt{\hat{P}[J]}=\frac{1}{V_{\mathcal{G}}} \int_{\mathcal{G}} d \mathbf{r} J^{*}(\mathbf{r}) e^{i k \hat{\mathbf{r}}_{0} \cdot \mathbf{r}} .
$$

Following the method of Lagrange, as explained in more detail in Appendix B, it is expedient to find the stationary function $J=J_{\text {st }}$ for the functional

$$
\hat{F}[J]=\sqrt{\hat{P}[J]}-\lambda_{1}(\hat{N}[J]-N)-\lambda_{2}(\hat{T}[J]-T),
$$

where $\lambda_{1}$ and $\lambda_{2}$ are real. With Eqs. (2.1), (2.14), and (3.1), variations of the current render $\hat{F}[J]$ stationary at $J=J_{\text {st }}$ if and only if $J_{\text {st }}$ satisfies the linear integral equation

$$
e^{i k \hat{\mathbf{r}}_{0} \cdot \mathbf{r}}-\lambda_{1} J_{\mathrm{st}}(\mathbf{r})-\frac{\lambda_{2}}{V_{\mathcal{G}}} \int_{\mathcal{G}} d \mathbf{r}^{\prime} K\left(\mathbf{r}, \mathbf{r}^{\prime}\right) J_{\mathrm{st}}\left(\mathbf{r}^{\prime}\right)=0 .
$$

The preceding equation is preferably recast in the form

$$
\widetilde{J}(\mathbf{r})+\frac{\alpha}{V_{\mathcal{G}}} \int_{\mathcal{G}} d \mathbf{r}^{\prime} K\left(\mathbf{r}, \mathbf{r}^{\prime}\right) \widetilde{J}\left(\mathbf{r}^{\prime}\right)=e^{i k \hat{\mathbf{r}}_{0} \cdot \mathbf{r}}
$$

where

$$
\widetilde{J}(\mathbf{r})=\lambda_{1} J_{\text {st }}(\mathbf{r}), \quad \alpha=\lambda_{2} / \lambda_{1}
$$

Alternatively, $\lambda_{1}$ can be set equal to 1 without loss of generality. Of course, $\int_{\mathcal{G}} d \mathbf{r}^{\prime}$ is interpreted as in Eq. (2.1).

Equation (3.4) is a Fredholm integral equation of the second kind [31], which, for a fixed frequency, gives $J(\mathbf{r})$ as a function of $\alpha$. Therefore, $C$ enters Eq. (3.4) as a parameter implicitly through $\alpha$. This equation has a unique admissible solution for any given $\alpha$, except for those finite negative $\alpha_{m}$ 's $\left(m=1,2, \ldots ;\left|\alpha_{m+1}\right| \geqslant\left|\alpha_{m}\right|\right)$ satisfying the homogeneous equation

$$
\mathcal{J}_{m}(\mathbf{r})+\frac{\alpha_{m}}{V_{\mathcal{G}}} \int_{\mathcal{G}} d \mathbf{r}^{\prime} K\left(\mathbf{r}, \mathbf{r}^{\prime}\right) \mathcal{J}_{m}\left(\mathbf{r}^{\prime}\right)=0, \quad m=1,2, \ldots
$$

It is noted in passing that the eigenfunctions $\mathcal{J}_{m}(m$ $=1,2, \ldots$ ) do not necessarily form a complete set; in other words, there can be admissible currents that are orthogonal to all $\mathcal{J}_{m}$. Such currents do not radiate [32,33], as discussed further in Appendix B.

The solution to Eq. (3.4) is an optimum current solving Problem II.2, i.e.,

$$
J_{\text {opt }}(\mathbf{r} ; \alpha)=\widetilde{J}(\mathbf{r} ; \alpha),
$$

provided

$$
\alpha>-\left|\alpha_{1}\right|
$$

All admissible currents solving Problem II.1 may be obtained via multiplication of $\widetilde{J}$ by an arbitrary constant. Notably, an integral equation resembling Eq. (3.4) has been given by Katsenelenbaum and Shalukhin [34] in their investigation of two-dimensional current distributions whose far field approximates a prescribed pattern with a given accuracy. When $\mathcal{G}$ is the interior of a closed curve in two dimensions, application of the Laplacian $\nabla^{2}$ to both sides of Eq. (3.4) shows that the optimum current satisfies Helmholtz's equation in $\mathcal{G}$, i.e.,

$$
\nabla^{2} J_{\text {opt }}(\mathbf{r})+k^{2} J_{\text {opt }}(\mathbf{r})=0, \quad \mathbf{r}=(x, y) \in \mathcal{G} .
$$

A special case of the integral equation (3.4) results when $\alpha=0$. The familiar current distribution

$$
\widetilde{J}_{0}(\mathbf{r}) \equiv \widetilde{J}(\mathbf{r} ; \alpha=0)=e^{i k \hat{\mathbf{r}}_{0} \cdot \mathbf{r}}
$$

is then trivially obtained. The preceding expression gives a current distribution of uniform magnitude everywhere in $\mathcal{G}$, which produces the optimum directivity by constructive interference. Such an arrangement of currents is conventional 
for achieving high directivity in a specified direction and is herein named "the reference case." The corresponding constraint and optimum directivity are

$$
C_{0}=D_{0}=T_{0}^{-1},
$$

where

$$
T_{0} \equiv \hat{T}\left[J=\widetilde{J}_{0}\right]=\frac{1}{V_{\mathcal{G}}^{2}} \int_{\mathcal{G}} d \mathbf{r} \int_{\mathcal{G}} d \mathbf{r}^{\prime} \widetilde{J}_{0}^{*}(\mathbf{r}) K\left(\mathbf{r}, \mathbf{r}^{\prime}\right) \widetilde{J}_{0}\left(\mathbf{r}^{\prime}\right)
$$

It follows that $D_{0}$ is always larger than $\varpi^{-1 / 2}$, where (see also Appendix B)

$$
\varpi=\varpi(k)=\frac{1}{V_{\mathcal{G}}^{2}} \int_{\mathcal{G}} d \mathbf{r} \int_{\mathcal{G}} d \mathbf{r}^{\prime}\left|K\left(\mathbf{r}, \mathbf{r}^{\prime}\right)\right|^{2}
$$

An asymptotic evaluation of this integral for $k \rightarrow \infty$ is carried out in Appendix D. In particular, this calculation verifies the expected result that $D_{0}$ is unbounded at high frequencies.

For $|\alpha|<\left|\alpha_{1}\right|$, an approximate expression for the optimum current can be formally obtained by the method of successive approximations, according to the scheme

$$
\begin{gathered}
\widetilde{J}^{(l+1)}(\mathbf{r})+\frac{\alpha}{V_{\mathcal{G}}} \int_{\mathcal{G}} d \mathbf{r}^{\prime} K\left(\mathbf{r}, \mathbf{r}^{\prime}\right) \widetilde{J}^{(l)}\left(\mathbf{r}^{\prime}\right)=e^{i k \hat{\mathbf{r}}_{0} \cdot \mathbf{r}}, \\
l=0, \quad 1, \ldots,
\end{gathered}
$$

where the zeroth-order approximation $\widetilde{J}^{(0)}$ is the reference current $\widetilde{J}_{0}$. This procedure yields

$$
\widetilde{J}^{(l)}(\mathbf{r})=g_{0}(\mathbf{r})-\alpha g_{1}(\mathbf{r})+\alpha^{2} g_{2}(\mathbf{r})+\cdots+(-1)^{l} \alpha^{l} g_{l}(\mathbf{r}),
$$

where

$$
\begin{gathered}
g_{0}(\mathbf{r})=\widetilde{J}_{0}(\mathbf{r}), \\
g_{l}(\mathbf{r})=\frac{1}{V_{\mathcal{G}}} \int_{\mathcal{G}} d \mathbf{r}^{\prime} K_{l}\left(\mathbf{r}, \mathbf{r}^{\prime}\right) \widetilde{J}_{0}\left(\mathbf{r}^{\prime}\right), \quad l=1,2, \ldots, \\
K_{1} \equiv K \\
K_{l}\left(\mathbf{r}, \mathbf{r}^{\prime}\right)=\frac{1}{V_{\mathcal{G}}} \int_{\mathcal{G}} d \mathbf{r}^{\prime \prime} K_{h}\left(\mathbf{r}, \mathbf{r}^{\prime \prime}\right) K_{l-h}\left(\mathbf{r}^{\prime \prime}, \mathbf{r}^{\prime}\right) \\
h=1,2, \quad \ldots, \quad l-1, \quad l=2,3, \quad \ldots
\end{gathered}
$$

For $l \rightarrow \infty$ and $|\alpha|<\left|\alpha_{1}\right|$, Eq. (3.15) converges to $\widetilde{J}$. The radius of convergence of the involved power series in $\alpha$ is $\left|\alpha_{1}\right|$, bounded below by $\varpi^{-1 / 2}$. As shown in Appendix D, the whole spectrum $\left\{a_{m}\right\}$ moves toward $-\infty$ at high frequencies. This in turn implies that the radius of convergence above is unbounded in $k$, depending on the frequency and the geometry of $\mathcal{G}$ in a nontrivial way. At high frequencies, Eqs. (3.14) - (3.17b) become advantageous to employ: For a prescribed geometry of the current-carrying region, an asymptotic evaluation of $g_{l}(\mathbf{r})(l=1,2, \ldots)$ can be car- ried out, leading to definite analytical representations for the optimum current. This task is planned for future works.

The integral equation (3.4) admits simple, closed-form solutions in terms of Fourier series in some special cases where region $\mathcal{G}$ is circularly symmetric. A few such cases of interest are examined in Appendix E.

\section{B. Discrete case}

The optimization of a discrete current distribution leads to a system of linear equations, which is the discrete analog of Eq. (3.4). Without further ado, the optimal amplitudes $\left\{I_{j}^{\text {(opt) }}\right\}_{j=1}^{M}$ are found to satisfy

$$
I_{j}^{(\mathrm{opt})}+\frac{\alpha}{M} \sum_{l=1}^{M} K\left(\mathbf{r}_{j}, \mathbf{r}_{l}\right) I_{l}^{(\mathrm{opt})}=e^{i k \hat{\mathbf{r}}_{0} \cdot \mathbf{r}_{j},} \quad j=1, \ldots, M,
$$

or, in matrix form,

$$
\left(\overline{\mathbf{1}}+\alpha \overline{\mathbf{K}}_{M}\right) \mathbf{I}^{(\mathrm{opt})}=\widetilde{\mathbf{I}}_{0},
$$

where $\overline{\mathbf{K}}_{M}=M^{-1}\left[K\left(\mathbf{r}_{j}, \mathbf{r}_{l}\right)\right]$ is a real and symmetric $M \times M$ matrix, $\quad \mathbf{I}^{(\mathrm{opt})}=\left[I_{l}^{(\mathrm{opt})}\right]$ and $\widetilde{\mathbf{I}}_{0}=\left[e^{i k \hat{\mathbf{r}}_{0} \cdot \mathbf{r}_{l}}\right]$ are $M \times 1$ column vectors, and $\overline{\mathbf{1}}$ is the unit $M \times M$ matrix. This equation was derived by Uzsoky and Solymár [23]. It admits a unique solution when $-\alpha^{-1}$ does not coincide with any one of the (positive) eigenvalues $-\xi_{m}^{-1}(m=1,2, \ldots, M)$ of matrix $\overline{\mathbf{K}}_{M}$, numbered in order of descending magnitude. This solution is an optimum current distribution provided (see also Appendix B)

$$
\alpha<-\left|\xi_{M}\right| \quad \text { or } \quad \alpha>-\left|\xi_{1}\right| \text {. }
$$

When $|\alpha|<\left|\xi_{1}\right|$, Eq. (3.18) is amenable to an approximation scheme analogous to Eq. (3.13).

\section{SOME PROPERTIES OF THE OPTIMUM DIRECTIVITY}

\section{A. Continuous case}

The constraint $C$ may not take arbitrary positive values. For continuous current distributions,

$$
C(\alpha) \geqslant\left|\alpha_{1}\right|,
$$

which is essentially Eq. (B13) of Appendix B. This minimum value is attained for $J=\mathcal{J}_{1}$. An expansion of the optimum current in the eigenfunctions $\mathcal{J}_{m}(m=1,2, \ldots)$ of Eq. (3.6) enables the derivation of a few properties of the relevant quantities (as outlined in Appendix C). Specifically,

$$
\begin{gathered}
\lim _{\alpha \rightarrow \infty} C(\alpha)=\infty, \\
\frac{d C(\alpha)}{d \alpha}>0,
\end{gathered}
$$

while 


$$
\frac{d D(\alpha)}{d \alpha}>0
$$

$$
\lim _{\alpha \rightarrow \infty} D(\alpha)=\infty
$$

where $D(\alpha)=\hat{D}[\widetilde{J}]$. The limit (4.3b) is a consequence of the speculative condition (C14) introduced in Appendix C. Note that letting $\alpha \rightarrow \infty$ is equivalent to solving the unconstrained optimization problem. With $\alpha=\alpha(C)$,

$$
\frac{d D}{d C}>0, \quad \lim _{C \rightarrow \infty} D(\alpha)=\infty,
$$

i.e., the optimal directivity is a monotonically increasing function of the imposed constraint and is unbounded for large values of the constraint. This result is expected, being in agreement with Oseen's Einstein needle radiation [1]. Furthermore,

$$
\begin{gathered}
\lim _{C \rightarrow \infty} \frac{d D}{d C}=0, \\
\frac{d^{2} D}{d C^{2}}<0,
\end{gathered}
$$

demonstrating that the larger the constraint is, the slower the rate of the directivity increase becomes. The total power radiated by a normalized optimum current becomes arbitrarily small as $C$ increases indefinitely. Equation (2.14) indicates that such a current varies extremely rapidly in $\mathbf{r}$.

\section{B. Discrete case}

In contrast, discrete current distributions are expected to introduce finite upper limits to the constraint $C=C(\alpha)$ and the directivity $D=D(\alpha)$ (as sketched in Appendix C). More precisely, for $-\left|\xi_{1}\right|<\alpha=\alpha(C)$,

$$
\begin{gathered}
\left|\xi_{1}\right|=C_{\min }=C\left(\alpha \rightarrow-\left|\xi_{1}\right|^{+}\right) \\
<C(\alpha) \leqslant C_{\infty} \equiv C(\alpha \rightarrow \infty)=\frac{\sum_{m=1}^{M}\left|\xi_{m}\right|^{2}\left|X_{m}\right|^{2}}{\sum_{m=1}^{M}\left|\xi_{m}\right|\left|X_{m}\right|^{2}} \\
\left|\xi_{1}\right|\left|X_{1}\right|^{2}=D\left(\alpha \rightarrow-\left|\xi_{1}\right|^{+}\right) \\
<D(\alpha) \leqslant D_{\infty} \equiv D(\alpha \rightarrow \infty), \\
D_{\infty}=\sum_{m=1}^{M}\left|\xi_{m}\right|\left|X_{m}\right|^{2}=\widetilde{\mathbf{I}}_{0}^{T} \overline{\mathbf{K}}_{M}^{-1} \widetilde{\mathbf{I}}_{0}, \\
\frac{d C(\alpha)}{d \alpha}>0, \quad \frac{d D}{d C}>0, \quad \frac{d^{2} D}{d C^{2}}<0,
\end{gathered}
$$

while for $\alpha<-\left|\xi_{M}\right|$,

$$
\begin{gathered}
C_{\infty} \leqslant C(\alpha)<C\left(\alpha \rightarrow-\left|\xi_{M}\right|^{-}\right)=C_{\max }=\left|\xi_{M}\right|, \\
\left|\xi_{M}\right|\left|X_{M}\right|^{2}=D\left(\alpha \rightarrow-\left|\xi_{M}\right|^{-}\right) \leqslant D(\alpha)<D_{\infty},
\end{gathered}
$$

$$
\frac{d C(\alpha)}{d \alpha}>0, \quad \frac{d D}{d C}<0, \quad \frac{d^{2} D}{d C^{2}}<0 .
$$

In the above,

$$
X_{m}=M^{-1} \sum_{j=1}^{M} I_{j}^{(m)} * e^{i k \hat{\mathbf{r}}_{0} \cdot \mathbf{r}_{j}}, \quad m=1,2, \ldots, M,
$$

$\mathbf{I}^{(m)}$ is the eigenvector of $\overline{\mathbf{K}}_{M}$ corresponding to $\xi_{m}$, and $\widetilde{\mathbf{I}}_{0}^{T}$ denotes the transpose of $\widetilde{\mathbf{I}}_{0}$. Note that the maximum optimal directivity $D_{\infty}$ corresponds to a constraint $C_{\infty}$ below the maximum value $C_{\max } . D_{\infty}$ is the solution to the unconstrained optimization problem, as introduced by Uzkov [35], and is produced by an optimum current

$$
\mathbf{I}^{(\text {opt })}=\operatorname{const} \times \sum_{m=1}^{\infty}\left|\xi_{m}\right| X_{m} \mathbf{I}^{(m)} .
$$

For constraints higher than $C_{\infty}$, the optimum directivity becomes a monotonically decreasing function of the constraint. It can be argued that such a behavior is accompanied by the appearance of an appreciable lobe about $-\hat{\mathbf{r}}_{0}$ in the far-field pattern. Accordingly, the curve $D=D(\alpha(C)) \equiv D_{\text {opt }}(C)$ exhibits analytic properties different from the case of continuous currents. For example, in the vicinity of the end point $C=C_{\max }$,

$$
D_{\text {opt }}(C)=\left|\xi_{M}\right|\left|X_{M}\right|^{2}+O\left(\sqrt{1-C / C_{\max }}\right),
$$

as proved in Appendix $\mathrm{C}$, and the slope of the curve becomes infinite there. A similar behavior at the lower endpoint $C$ $=C_{\min }$ is also uncovered in the continuous case. These results indicate that the analytic continuation of $D_{\text {opt }}(C)$ from $\Delta_{C}=\left[C_{\min }, C_{\max }\right]$ to complex values of $C$ encounters at least two branch points at $C=C_{\min }$ and $C=C_{\max }$. In the first Riemann sheet, $D_{\text {opt }}\left(C \in \Delta_{C}\right)$ is the optimum directivity. Encircling the point $C=C_{\max }$ once results in entering an adjacent Riemann sheet, where $D_{\text {opt }}\left(C \in \Delta_{C}\right)$ is presumably a minimum or a saddle point.

A close examination of the spectrum of $\overline{\mathbf{K}}_{M}$ verifies that high optimum directivities are favored by large $M$ and electrically small spacings $\left|\mathbf{r}_{i}-\mathbf{r}_{j}\right|(i \neq j)$. Of primary interest are optimum currents that produce directivities moderately larger than the reference value $D_{0}$ with the corresponding constraints only slightly above $C_{0}$. This calls for studying Eq. (3.4) in specific geometries of the current-carrying region $\mathcal{G}$ for positive values of $\alpha$ that validate expansion (3.15). Similar considerations hold in regard to Eq. (3.19).

\section{DISCUSSION}

The foregoing analysis admits several generalizations, but also points to certain open questions. In particular, note the following.

(i) If the polarization of the continuous current distribution $\mathbf{J}(\mathbf{r})$ is also free to vary, optimization of the current vector yields essentially two independent linear integral equations: one for the component parallel to $\mathbf{r}_{0}$ and one for a component normal to $\mathbf{r}_{0}$. The former equation is homoge- 
neous and has only the zero solution (for $\alpha>0$ ), while the latter is Eq. (3.4). This situation is totally different when the maximized quantity is the power radiated through a solid angle (say, centered at $\mathbf{r}_{0}$ ).

(ii) When $\mathcal{G}$ consists of $M_{d}$ disjoint regions $\mathcal{G}_{1}, \mathcal{G}_{2}, \ldots, \mathcal{G}_{M_{d}} \quad\left(\mathcal{G}_{i} \cap \mathcal{G}_{j}=\emptyset\right.$ for any $i \neq j$; $i, j=1,2, \ldots, M_{d}$ ), then Eq. (3.4) reads

$$
\begin{aligned}
& \widetilde{J}(\mathbf{r})+\frac{\alpha}{V_{\mathcal{G}}}\left[\int_{\mathcal{G}_{i}} d \mathbf{r}^{\prime} K\left(\mathbf{r}, \mathbf{r}^{\prime}\right) \widetilde{J}\left(\mathbf{r}^{\prime}\right)+\sum_{\substack{j=1 \\
(j \neq i)}}^{M_{d}} \int_{\mathcal{G}_{j}} d \mathbf{r}^{\prime} K\left(\mathbf{r}, \mathbf{r}^{\prime}\right) \widetilde{J}\left(\mathbf{r}^{\prime}\right)\right] \\
& =e^{i k \hat{\mathbf{r}_{0}} \cdot \mathbf{r}}, \quad \mathbf{r} \in \mathcal{G}_{i} .
\end{aligned}
$$

(iii) An important variant of Problem II.1 is the following. Problem V.1. Given a current-carrying region $\mathcal{G}$ (or $\mathbf{r}_{j}$, $j=1, \ldots, M$, in the discrete case) and $\hat{\mathbf{r}}_{0}$, determine admissible continuous current distributions $J(\mathbf{r})=J_{\text {opt }}(\mathbf{r})$ (or discrete $\left\{I_{j}=I_{j}^{(\mathrm{opt})}\right\}$ ) that maximize $\hat{D}[J]$ (or $\hat{D}[\mathbf{I}]$ ) for $\hat{C}[J] \leqslant C$ (or $\hat{C}[\mathbf{I}] \leqslant C)$.

According to Sec. IV, this problem is equivalent to Problem II.1 for continuous current distributions and any (permissible) $C \geqslant\left|\alpha_{1}\right|$. In contrast, such an equivalence is ensured for discrete currents only when $C \leqslant C_{\infty}$. For $C>C_{\infty}$, the solution to Problem V.1 is always given by Eq. (4.13), as the solution to the unconstrained optimization problem.

(iv) With the norm defined by Eq. (2.1), threedimensional volume currents that maximize $D=P / T$ under fixed $C=N / T$ are discontinuous across the boundary $\mathcal{S}_{\mathcal{G}}$ of region $\mathcal{G}$. Conservation of charge demands that the discontinuity in their component normal to $\mathcal{S}_{\mathcal{G}}$ be accompanied by surface charge distributed on $\mathcal{S}_{\mathcal{G}}$. A moment's reflection leads to the idea that this possibility could be eliminated mathematically by solving a similar optimization problem in some subspace of all currents satisfying Eq. (2.1); for instance, $N$ could be redefined by properly adding squares of space derivatives of current density components under the integral sign. Such a modification would result in an integrodifferential equation for an optimum current density of fixed polarization, which, if supplemented with the appropriate boundary condition, can furnish a solution continuous across $\mathcal{S}_{\mathcal{G}}$. Formulation of a physically reasonable problem of this type is left as an open question for future work.

(v) Issues related to bandwidth are also left as open questions: It is difficult (if not impossible) to make quantitative statements about bandwidths of current-carrying regions with directivities not much larger than $D_{0}$. To make such statements, one must first specify the method of exciting (or approximating) the idealized current distribution.

(vi) The properties of current-carrying regions with directivities larger than $D_{0}$ are frequency dependent. Previous studies of highly directive radiating systems have tended to focus on electrically small structures [9]. As evidenced by recent studies of electrically large, resonant, closed-loop antennas $[15,17,36]$, electrically large structures are particularly interesting. The formulation developed here is especially suitable for finding bounds on the radiating efficiencies of such structures. These bounds are sharper than bounds originating from methods that deal with the fields on a surrounding sphere by leaving the radiating structure unspecified.

(vii) In addition to obtaining bounds for the aforementioned structures, it is of interest to compare the optimum currents obtained from the present formulation to the current distributions in resonant closed loops [17]. Are these similar (at least for some ranges of $C$ and for some frequencies and shapes) to one another? If the answer to this question is proved to be positive, it may be possible to excite the optimum currents in noncircular closed loops. These considerations call for a study of optimum, continuous current distributions in closed loops.

(viii) The present approach provides a suitable analytical tool for studying highly directive continuous currents for $C$ 's above the $C_{0}$ of the uniform distribution. For electrically large structures, a challenging task is to describe approximately such currents uniformly in $0<\alpha<\infty$. This investigation may shed light on the conditions under which the Einstein needle radiation becomes a theoretical possibility. In this case, the currents are, in general, rapidly varying functions of $\mathbf{r}$ in region $\mathcal{G}$.

(ix) Though not mathematically compelling, it seems physically reasonable to presume that Eqs. (4.4)-(4.5b) imply a nearly logarithmic dependence of the optimum $D_{\text {opt }}(C)$ on $C$, when the latter approaches infinity. Intuitively, it is expected that the leading asymptotic behavior of $D_{\text {opt }}(C)$ as $C \rightarrow \infty$ is universal in two dimensions, being inherent to some "wide" class of current-carrying regions $\mathcal{G}$. This behavior is in turn determined by the leading asymptotic form of $\left|\alpha_{m}\right|$ as $m \rightarrow \infty$ and calls for an investigation of the homogeneous integral equation for large $\alpha$. These considerations can possibly be extended to three-dimensional planar regions, with $\mathbf{r}_{0}$ lying in the plane of $\mathcal{G}$.

(x) The solution to Eq. (3.4) can be given in a simple closed form in the case of the two-dimensional circular current distribution. Although the circle does not naturally lend itself to establishing highly directive fields and the conjecture attempted in (vii) above does not hold in this case, it is desirable to study the circular closed loop first in detail.

\section{CONCLUSIONS}

Starting with Maxwell's equations, the present work develops a self-contained theory of optimally directive currents in two and three dimensions and studies analytically some of the physical implications. More precisely, maximization of the directivity in the far field of an idealized radiating system, under the constraint of a fixed ratio of the current norm squared over the total radiated power, is shown to lead to a linear integral equation for the optimum continuous current. In this equation, the dependence on the constraint enters implicitly through the Lagrange multiplier $\alpha$. This integral equation is recognized as a tool for studying analytically currents that produce directivities larger than the uniform distribution (reference case), where $\alpha$ assumes positive values. One range of $\alpha$ that is of interest involves small increases in directivity above the reference case. The optimum current may then be calculated by use of iteration on the integral equation. Another range of physical interest corresponds to the extreme case in which $\alpha$ approaches infinity 
and currents reversing rapidly in space give rise to the Einstein needle radiation.

A close examination of Eqs. (3.4) and (3.19) for the optimum continuous and discrete currents reveals that the general properties of the optimum directivity, currents, and fields are related to the eigenvalues and eigenfunctions of the corresponding kernel or matrix, respectively. For discrete currents, the number of the eigenvalues is finite, resulting in a (finite) maximum directivity for a constraint smaller than its maximum (finite) value. When the constraint approaches its maximum value, the slope of the optimum directivity exhibits a singular behavior as a function of the constraint. For continuous currents, the eigenvalues become infinite in number and the optimum directivity tends to infinity monotonically, with an ultimately decreasing rate of increase.

The present formulation is applicable to any size of $\mathcal{G}$. However, it is more advantageous for the study of the interesting case of electrically large structures: First, they allow for simplifying approximations in the derived equations, such as the iteration scheme (3.14). This is because the wavelength is small compared to the maximum linear dimension of $\mathcal{G}$ and asymptotic methods become effective, as implied by the analysis of Appendix D. Second, they may provide a link to other recent studies and experiments [17], illuminating the difficult problem of realization of currents. We plan to apply the theoretical framework discussed here to electrically large systems in future works.

\section{ACKNOWLEDGMENTS}

The authors are greatly indebted to Professor Ronold W. P. King for his insightful comments and suggestions. D.M. wishes to thank Dr. Jianmei Wang for a useful discussion. G.F. thanks Dr. Arje Nachman for bringing Ref. [27] to his attention. This work was supported in part by the Joint Services Electronics Program under Grant No. N00014-89-J1023 with Harvard University and in part by AFOSR support of Rome Laboratory.

\section{APPENDIX A: EVALUATION OF $K\left(r, \mathbf{r}^{\prime}\right)$}

An integral expression for the three-dimensional kernel entering Eq. (2.14) reads

$$
\begin{aligned}
K^{(3)}\left(\mathbf{r}_{1}, \mathbf{r}_{2}\right)= & \frac{1}{4 \pi} \int_{(4 \pi)} d \Omega \sin ^{2} \theta e^{i k \hat{\mathbf{r}} \cdot \mathbf{R}} \\
& \mathbf{R}=\mathbf{r}_{1}-\mathbf{r}_{2}, \quad \mathbf{r}_{1}, \mathbf{r}_{2} \in \mathcal{G}
\end{aligned}
$$

where integration is understood over $\hat{\mathbf{r}}[\mathbf{r}=(x, y, z)]$, and $\theta$ is the angle between $\hat{\mathbf{z}}$ and $\hat{\mathbf{r}}$. Let $\left(x^{\prime}, y^{\prime}, z^{\prime}\right)$ be a rotated Cartesian coordinate system such that $\hat{\mathbf{z}}^{\prime}=\hat{\mathbf{R}}$ and $\hat{\mathbf{x}}^{\prime}$ is coplanar with $\hat{\mathbf{z}}$ and $\mathbf{R}$. Let $\chi$ denote the angle between $\mathbf{R}$ and $\hat{\mathbf{z}}$ $(\hat{\mathbf{z}} \cdot \hat{\mathbf{R}}=\cos \chi)$. With the introduction of the spherical coordinate system $(r, \beta, \gamma)$ associated with the primed system by convention, Eq. (A1) is cast in the form

$$
\begin{aligned}
K^{(3)}\left(\mathbf{r}_{1}, \mathbf{r}_{2}\right)= & \frac{1}{4 \pi} \int_{0}^{2 \pi} d \gamma \int_{0}^{\pi} d \beta \sin \beta \\
& \times\left[1-\sin ^{2} \chi \sin ^{2} \beta \cos ^{2} \gamma-\cos ^{2} \chi \cos ^{2} \beta\right. \\
& -\sin 2 \chi \sin \beta \cos \beta \cos \gamma] e^{i k R \cos \beta} \\
= & \frac{1}{4}\left[\left(2-\sin ^{2} \chi\right) \int_{-1}^{1} d \xi e^{i k R \xi}\right. \\
& \left.+\left(\sin ^{2} \chi-2 \cos ^{2} \chi\right) \int_{-1}^{1} d \xi \xi^{2} e^{i k R \xi}\right] \\
= & \sin ^{2} \chi \frac{\sin (k R)}{k R} \\
& +\left(1-3 \cos ^{2} \chi\right)\left[\frac{\cos (k R)}{(k R)^{2}}-\frac{\sin (k R)}{(k R)^{3}}\right],
\end{aligned}
$$

which is Eq. (2.15).

In two dimensions, the corresponding integral reads

$$
K^{(2)}\left(\mathbf{r}_{1}, \mathbf{r}_{2}\right)=\frac{1}{2 \pi} \int_{0}^{2 \pi} d \gamma e^{i k R \cos \gamma} .
$$

This is easily identified with Eq. (2.22).

\section{APPENDIX B: DETAILED STUDY OF THE MAXIMIZATION PROBLEM}

This appendix investigates in some detail the maximization of the power-flux functional $\hat{P}[J]$. In particular, it provides a description of the optimum current in terms of the appropriate eigenfunctions.

\section{Continuous case}

The kernel $K\left(\mathbf{r}, \mathbf{r}^{\prime}\right)$ of the integral equation (3.4) is Hermitian. It follows that the various nonzero solutions $\mathcal{J}_{m}$ ( $m$ $=1,2,3, \ldots)$ of the homogeneous counterpart of Eq. (3.4), viz.,

$$
J(\mathbf{r})+\alpha K_{\mathrm{op}} J(\mathbf{r})=0, \quad K_{\mathrm{op}} J(\mathbf{r}) \equiv \frac{1}{V_{\mathcal{G}}} \int_{\mathcal{G}} d \mathbf{r}^{\prime} K\left(\mathbf{r}, \mathbf{r}^{\prime}\right) J\left(\mathbf{r}^{\prime}\right),
$$

are orthogonal, corresponding to real eigenvalues $\alpha_{m}$ numbered in order of ascending magnitude $\left(\left|\alpha_{m}\right| \rightarrow \infty\right.$ for $m$ $\rightarrow \infty$ ), with eigenvalues of multiplicity $l>1$ being repeated $l$ times. The $\alpha_{m}$ are negative because $\hat{T}[J] \geqslant 0$ for any admissible current $J$, dictating that $K\left(\mathbf{r}, \mathbf{r}^{\prime}\right)$ is a positive kernel. The set $\left\{\mathcal{J}_{m}\right\}$ is not necessarily complete [31]. It is noted in passing that $K\left(\mathbf{r}, \mathbf{r}^{\prime}\right)$ is smooth in $\left(\mathbf{r}, \mathbf{r}^{\prime}\right) \in \mathcal{G} \times \mathcal{G}$, i.e., it has continuous partial derivatives of any order there. The kernel decomposition

$$
K\left(\mathbf{r}, \mathbf{r}^{\prime}\right)=\sum_{m=1}^{\infty} \frac{\mathcal{J}_{m}^{*}(\mathbf{r}) \mathcal{J}_{m}\left(\mathbf{r}^{\prime}\right)}{\left|\alpha_{m}\right|}
$$

entails the rather simple formula 
$\sum_{m=1}^{\infty} \frac{1}{\left|\alpha_{m}\right|}=\frac{1}{V_{\mathcal{G}}} \int_{\mathcal{G}} d \mathbf{r} K(\mathbf{r}, \mathbf{r})= \begin{cases}2 / 3 & \text { in three dimensions } \\ 1 & \text { in two dimensions }\end{cases}$

In the Dirac notation

$$
\langle g, h\rangle=\frac{1}{V_{\mathcal{G}}} \int_{\mathcal{G}} d \mathbf{r} g *(\mathbf{r}) h(\mathbf{r}),
$$

the normalization of $\mathcal{J}_{m}$ so that $\left\langle\mathcal{J}_{m}, \mathcal{J}_{m^{\prime}}\right\rangle=\delta_{m m^{\prime}}$ and the use of Eq. (B1) give a formal representation for the solution $\widetilde{J}$ of Eq. (3.4):

$$
\widetilde{J}(\mathbf{r})=\widetilde{J}_{0}(\mathbf{r})-\alpha \sum_{m=1}^{\infty} \frac{1}{\left|\alpha_{m}\right|} \frac{A_{m}}{1+\alpha /\left|\alpha_{m}\right|} \mathcal{J}_{m}(\mathbf{r}) .
$$

In the above,

$$
A_{m}=\left\langle\mathcal{J}_{m}, \widetilde{J}_{0}\right\rangle, \quad \sum_{m=1}^{\infty}\left|A_{m}\right|^{2}=1, \quad \widetilde{J}_{0}(\mathbf{r})=e^{i k \hat{\mathbf{r}}_{0} \cdot \mathbf{r}} .
$$

A close examination of Eq. (3.4) reveals that $\widetilde{J}(\mathbf{r})$ is a smooth function of $\mathbf{r}$, where $\mathbf{r}$ lies inside region $\mathcal{G}(\mathbf{r} \in \mathcal{G})$. This is also true for each $\mathcal{J}_{m}(\mathbf{r})$. If $\mathcal{G}$ is a curve, differentiation is understood with respect to the arc length $s$. Series (B5) converges uniformly in $\mathbf{r} \in \mathcal{G}$.

For the purpose of showing that Eq. (B5) indeed maximizes $\hat{P}[J],\left\{\mathcal{J}_{m}\right\}_{m=1}^{\infty}$ is complemented with an orthonormal set $\left\{\breve{\mathcal{J}}_{p}\right\}_{p=1}^{L}$, where $L$ can possibly be infinite $(L \rightarrow \infty)$, so that their union forms a complete set and

$$
\left\langle\mathcal{J}_{m}, \breve{\mathcal{J}}_{p}\right\rangle=0, \quad m=1,2, \ldots, \quad p=1,2, \ldots, L .
$$

Then any admissible current distribution $J(\mathbf{r})$ can be expanded in a Fourier-type series

$$
J(\mathbf{r})=\sum_{m=1}^{\infty} a_{m} \mathcal{J}_{m}(\mathbf{r})+\sum_{p=1}^{L} b_{p} \breve{\mathcal{J}}_{p},
$$

where

$$
\begin{aligned}
a_{m} & =\left\langle\mathcal{J}_{m}, J\right\rangle, \quad m=1,2,3, \ldots, \\
b_{p} & =\left\langle\breve{\mathcal{J}}_{p}, J\right\rangle, \quad p=1,2, \ldots, L .
\end{aligned}
$$

It follows that

$$
\begin{gathered}
\hat{N}[J]=\langle J, J\rangle=\sum_{m=1}^{\infty}\left|a_{m}\right|^{2}+\sum_{p=1}^{L}\left|b_{p}\right|^{2}, \\
\hat{T}[J]=\left\langle J, K_{\mathrm{op}} J\right\rangle=\sum_{m=1}^{\infty} \frac{\left|a_{m}\right|^{2}}{\left|\alpha_{m}\right|} .
\end{gathered}
$$

Consequently,

$$
\hat{T}[J] \leqslant\left|\alpha_{1}\right|^{-1} \quad \text { if }\langle J, J\rangle=1,
$$

which implies that $\left|\alpha_{1}\right|$ is the minimum value of the constraint $\hat{C}[J]$, attained with the normalized current $J=\mathcal{J}_{1}$. By use of Eq. (B1),

$$
\frac{1}{\left|\alpha_{1}\right|^{2}}<\varpi(k), \quad \varpi(k)=\frac{1}{V_{\mathcal{G}}^{2}} \int_{\mathcal{G}} d \mathbf{r} \int_{\mathcal{G}} d \mathbf{r}^{\prime}\left|K\left(\mathbf{r}, \mathbf{r}^{\prime}\right)\right|^{2} .
$$

In the space orthogonal to $\mathcal{J}_{1}, \ldots, \mathcal{J}_{m-1}$, similar considerations hold for each of the other eigenvalues $\alpha_{m}(m \geqslant 2)$. In particular,

$$
\left\langle\mathcal{J}_{m}, K_{\mathrm{op}} \mathcal{J}_{m}\right\rangle=\frac{1}{\left|\alpha_{m}\right|}, \quad \lim _{m \rightarrow \infty}\left\langle\mathcal{J}_{m}, K_{\mathrm{op}} \mathcal{J}_{m}\right\rangle=0,
$$

suggesting that $\hat{C}[J]$ does not have a (finite) upper bound.

According to Eq. (B12), if currents $\breve{\mathcal{J}}_{p}$ exist, they do not radiate. Due to the analyticity of the electromagnetic field in free space, it is concluded that the field produced by each $\breve{\mathcal{J}}_{p}$ vanishes identically outside of $\mathcal{G}$ [37]. Hence

$$
\left\langle\widetilde{J}_{0}, \breve{\mathcal{J}}_{p}\right\rangle=0, \quad p=1,2, \ldots, L .
$$

In the case of two-dimensional current distributions, it is of some interest to note that when region $\mathcal{G}$ is a closed loop, a single "nonradiating" current $\breve{\mathcal{J}}_{1}$ exists $(L=1)$ only at the discrete spectrum of frequencies that solve the internal Dirichlet problem. No such current exists if $\mathcal{G}$ is an open curve. When $\mathcal{G}$ is the interior of a closed loop, $\left\{\breve{\mathcal{J}}_{p}\right\}$ is always infinite, as is illustrated for the circular disk in Appendix E.

Accordingly, the scalar field at $\hat{\mathbf{r}}_{0}$ is given by

$$
\hat{\Psi}[J]=\sum_{m=1}^{\infty} a_{m} A_{m}^{*} .
$$

Without loss of generality, $A_{m}$ are assumed to be real $\left(A_{m}\right.$ $\left.=A_{m}^{*}\right)$.

Since $\hat{N}[J]$ and $\hat{T}[J]$ are independent of the phases $\operatorname{Arg} a_{m}$ and $\operatorname{Arg} b_{p}$, the latter can be freely chosen to $\operatorname{maximize}|\hat{\Psi}[J]|=\sqrt{\hat{P}[J]}$. A necessary condition for maximum real $\hat{\Psi}[J]$ is

$$
a_{m} A_{m} \geqslant 0, \quad m=1,2,3, \ldots,
$$

while it is mathematically convenient to take $b_{p}$ to be real $\left(b_{p}=b_{p}^{*}\right)$. The task is thus assigned to apply the method of Lagrange multipliers in order to maximize the real functional

$$
\hat{\Psi}\left[\left\{a_{m}\right\},\left\{b_{p}\right\}\right]=\psi\left(\hat{\mathbf{r}}_{0}\right)=\sum_{m=1}^{\infty} a_{m} A_{m},
$$

by keeping $\hat{N}[J]=N$ and $\hat{T}[J]=T$ fixed. For finite sets $\left\{a_{m}\right\}_{m=1}^{n_{1}}$ and $\left\{b_{p}\right\}_{p=1}^{n_{2}}\left(n_{2} \leqslant L\right), n=n_{1}+n_{2}$, and sufficiently large $n_{1}$, Eqs. (B11) and (B12) together define a smooth $n-2$ manifold $\mathcal{M}_{n-2}$ as the intersection of an $n-1$ sphere with an $n-1$ ellipsoid (for $n_{2}=0$ ), or an $n-1$ cylinder (for $n_{2} \geqslant 1$ ). The geometry is easily visualized for $n=3$, where $\mathcal{M}_{n-2}=\mathcal{M}_{1}$ consists of two smooth closed curves. Since 
any linear function can be described as a set of parallel hyperplanes, maximization or minimization in $\mathcal{M}_{n-2}$ of the function

$$
\Psi_{n}\left(a_{1}, \ldots, a_{n_{1}}, b_{1}, \ldots, b_{n_{2}}\right)=\sum_{m=1}^{n_{1}} a_{m} A_{m}
$$

occurs at points where the corresponding hyperplanes are tangent to $\mathcal{M}_{n-2}$. Clearly, the method of Lagrange multipliers yields all desired maxima or minima of $\Psi_{n}$. By relaxing the requirements of mathematical rigor, these considerations can be extended to infinite $n(n \rightarrow \infty)$. Accordingly, the optimum amplitudes $\left\{a_{m}^{(\mathrm{opt})}\right\}$ and $\left\{b_{p}^{(\mathrm{opt})}\right\}$ are stationary points of

$$
\begin{aligned}
\hat{F}[J]= & \hat{\Psi}[J]-\nu_{1}(\hat{N}[J]-N) \\
& -\nu_{2}(\hat{T}[J]-T), \text { for } \nu_{1}, \nu_{2} \text { real. }
\end{aligned}
$$

The first variation of $\hat{F}[J]$ vanishes when

$$
2 \nu_{1}\left(1+\nu /\left|\alpha_{m}\right|\right) a_{m}^{(\mathrm{opt})}=A_{m}, \quad 2 \nu_{1}\left|b_{p}^{(\mathrm{opt})}\right|=0, \quad \nu=\nu_{2} / \nu_{1} \text {. }
$$

$\hat{\Psi}$ is evidently maximized only when $\nu_{1}$ is non-negative and $\nu>-\left|\alpha_{1}\right|$. In the spirit of Sec. III, $\nu_{1}$ is set equal to $1 / 2$, leading to

$$
a_{m}^{(\mathrm{opt})}=\frac{A_{m}}{1+\nu /\left|\alpha_{m}\right|} .
$$

Equation (B5) is then recovered with $\alpha=\nu$ :

$$
J_{\text {opt }}(\mathbf{r})=\sum_{m=1}^{\infty} \frac{A_{m}}{1+\alpha /\left|\alpha_{m}\right|} \mathcal{J}_{m}(\mathbf{r})
$$

Therefore, for $\alpha>-\left|\alpha_{1}\right|$, the unique admissible solution to the integral equation (3.4) is an optimum current distribution.

A close examination of Eq. (B22) reveals that the method of Lagrange multipliers furnishes a maximum only for $\alpha>$ $-\left|\alpha_{1}\right|$. In particular, for $\alpha \rightarrow-\left|\alpha_{1}\right|^{+}$,

$$
C(\alpha) \rightarrow\left|\alpha_{1}\right|^{+} \text {. }
$$

For $C=\left|\alpha_{1}\right|$, direct use of the definition for the constraint $C$ gives a current proportional to the eigenfunction $\mathcal{J}_{1}(\mathbf{r})$. The limit $\alpha \rightarrow \infty$ is equivalent to taking $\nu_{1}=0$ ab initio; therefore, it gives the solution to the unconstrained optimization problem.

By virtue of Eqs. (B11), (B12), and (B19),

$$
\begin{gathered}
P(\alpha)=\hat{P}[\widetilde{J}]=\left(\sum_{m=1}^{\infty} \frac{\left|A_{m}\right|^{2}}{1+\alpha /\left|\alpha_{m}\right|}\right)^{2}, \\
N(\alpha)=\hat{N}[\widetilde{J}]=\sum_{m=1}^{\infty} \frac{\left|A_{m}\right|^{2}}{\left(1+\alpha /\left|\alpha_{m}\right|\right)^{2}}, \\
T(\alpha)=\hat{T}[\widetilde{J}]=\sum_{m=1}^{\infty} \frac{1}{\left|\alpha_{m}\right|} \frac{\left|A_{m}\right|^{2}}{\left(1+\alpha /\left|\alpha_{m}\right|\right)^{2}}, \\
C(\alpha)=N(\alpha) / T(\alpha), \quad D(\alpha)=P(\alpha) / T(\alpha) .
\end{gathered}
$$

Equations (B3) and (B6) ensure absolute and uniform-in- $\alpha$ convergence of Eqs. (B24) and (B26)-(B29) for any finite complex $\alpha \neq \alpha_{m}(m=1,2,3, \ldots)$ and $\mathbf{r}$ inside region $\mathcal{G}$.

Let $\Gamma$ denote any finite closed contour in the complex $\alpha$ plane not enclosing or passing through any of the points $\alpha_{m}$. Integration of (B24) along $\Gamma$ yields

$$
\oint_{\Gamma} d \alpha J_{\mathrm{opt}}(\mathbf{r} ; \alpha)=\sum_{m=1}^{\infty} A_{m} \mathcal{J}_{m}(\mathbf{r}) \oint_{\Gamma} \frac{d \alpha}{1+\alpha /\left|\alpha_{m}\right|}=0 .
$$

Hence $J_{\text {opt }}(\mathbf{r} ; \alpha)$ is a meromorphic function of $\alpha$ for fixed $\mathbf{r}$ inside $\mathcal{G}$, with simple poles at $\alpha=\alpha_{m}(m=1,2,3, \ldots)$. Similarly, $P(\alpha), N(\alpha)$, and $T(\alpha)$ are meromorphic functions of $\alpha$, with double poles at $\alpha=\alpha_{m}$.

By virtue of the binomial expansion

$$
\begin{aligned}
& \left(1+\frac{\alpha}{\left|\alpha_{m}\right|}\right)^{-q}=\sum_{l=0}^{\infty} \frac{(-1)^{l}}{l !} \frac{(l+q-1) !}{(q-1) !}\left(\frac{\alpha}{\left|\alpha_{m}\right|}\right)^{l}, \\
& \text { for } q \text { an integer }>0,
\end{aligned}
$$

which is valid for $|\alpha|<\left|\alpha_{m}\right|$, power series in $\alpha$ of all optimum quantities can be derived. For $|\alpha|<\left|\alpha_{1}\right|$, the optimum current is expanded as

$$
J_{\text {opt }}(\mathbf{r} ; \alpha)=\sum_{l=0}^{\infty}(-1)^{l} \alpha^{l} g_{l}(\mathbf{r})
$$

where

$$
g_{l}(\mathbf{r})=\sum_{m=1}^{\infty} A_{m}\left|a_{m}\right|^{-l} \mathcal{J}_{m}(\mathbf{r}), \quad l=0,1,2, \ldots,
$$

given in integral form (for $l \geqslant 1$ ) by Eq. (3.16b).

\section{Discrete case}

Given the eigenvalues $\left|\xi_{m}\right|^{-1}$ and eigenvectors $\mathbf{I}^{(m)}(m$ $=1,2, \ldots, M)$ of the real, symmetric, and positive definite matrix $\overline{\mathbf{K}}_{M}$ of Eq. (3.19) in the $M$-dimensional vector space, along with the definition (4.12), the foregoing analysis for continuous current distributions can be basically applied to discrete distributions. Heuristically speaking, the major replacements are

$$
\frac{1}{V_{\mathcal{G}}} \int_{\mathcal{G}} d \mathbf{r}^{\prime} \rightarrow M^{-1} \sum_{l=1}^{M}, \quad \mathbf{r} \in \mathcal{G} \rightarrow\left\{\mathbf{r}_{j}\right\}_{j=1}^{M}, \quad \sum_{m=1}^{\infty} \rightarrow \sum_{m=1}^{M}
$$

and

$$
\begin{gathered}
\mathcal{J}_{m}(\mathbf{r}) \rightarrow I_{l}^{(m)}, \quad A_{m} \rightarrow X_{m}, \quad \alpha_{m} \rightarrow \xi_{m} \\
(l, m=1,2, \quad \ldots, M)
\end{gathered}
$$

Two points need to be emphasized here. 
(i) Since the spectrum of $\overline{\mathbf{K}}_{M}$ is finite, the permissible range of $\nu=\alpha$ in an equation analogous to Eq. (B22) now extends to $\left(-\infty,-\left|\xi_{M}\right|\right)$ and the corresponding $\nu_{1}$ becomes negative.

(ii) $\mathbf{I}^{(m)}$ always form a complete set. Therefore, the concept of nonradiating currents does not apply.

\section{APPENDIX C: PROPERTIES OF THE OPTIMUM QUANTITIES}

In this appendix, some general properties of the quantities involved in the optimization problem are derived.

\section{Continuous case}

It is shown that $C(\alpha)$ does not have a (finite) upper bound. For any fixed $A \gg 1$ and any positive $\epsilon \ll 1$, there exists an $m_{0}=m_{0}(A) \gg 1$ such that

$$
\left|\alpha_{m}\right|>A, \quad m>m_{0}
$$

while

$$
\sum_{m=1}^{m_{0}} \frac{1}{\left|\alpha_{m}\right|} \frac{\left|A_{m}\right|^{2}}{\left(1+\alpha /\left|\alpha_{m}\right|\right)^{2}}<\frac{\epsilon}{A}
$$

provided $\alpha=\alpha(\epsilon)$ is sufficiently large. Consequently,

$$
C(\alpha) \geqslant A \frac{\sum_{m=m_{0}+1}^{\infty} \frac{\left|A_{m}\right|^{2}}{\left(1+\alpha /\left|\alpha_{m}\right|\right)^{2}}}{\epsilon+\sum_{m=m_{0}+1}^{\infty} \frac{\left|A_{m}\right|^{2}}{\left(1+\alpha /\left|\alpha_{m}\right|\right)^{2}} .}
$$

Since $\epsilon$ can be chosen arbitrarily small, Eq. (4.2a) follows.

From Eqs. (B26)-(B28) it is inferred that $P(\alpha), N(\alpha)$, and $T(\alpha)$ are monotonically decreasing functions of $\alpha$. Obviously,

$$
\begin{gathered}
\sqrt{P(\alpha)}-N(\alpha)=\alpha T(\alpha), \\
{[\sqrt{P(\alpha)}]^{\prime}=-T(\alpha),}
\end{gathered}
$$

where the prime denotes differentiation with respect to $\alpha$. It is of some interest to note that by inspection of these equations the optimum scalar field at $\hat{\mathbf{r}}=\hat{\mathbf{r}}_{0}$ equals

$$
\psi\left(\hat{\mathbf{r}}_{0} ; \alpha\right)=\sqrt{P(\alpha)}=\frac{1}{\alpha} \int_{0}^{\alpha} d \xi N(\xi),
$$

i.e., it is the mean value of $N(\alpha)$ over the "past" positive Lagrange multipliers $\alpha$. After some straightforward algebra,

$$
\begin{aligned}
C^{\prime}(\alpha)= & -\frac{1}{T(\alpha)^{2}}\left[2 T(\alpha)^{2}+\sqrt{P(\alpha)} T^{\prime}(\alpha)\right] \\
= & \frac{2}{T(\alpha)^{2}}\left[\left(\sum_{m=1}^{\infty} \frac{\left|A_{m}\right|^{2}}{1+\alpha /\left|\alpha_{m}\right|}\right)\right. \\
& \times\left(\sum_{m=1}^{\infty} \frac{1}{\left|\alpha_{m}\right|^{2}} \frac{\left|A_{m}\right|^{2}}{\left(1+\alpha /\left|\alpha_{m}\right|\right)^{3}}\right)
\end{aligned}
$$

$$
\left.-\left(\sum_{m=1}^{\infty} \frac{1}{\left|\alpha_{m}\right|} \frac{\left|A_{m}\right|^{2}}{\left(1+\alpha /\left|\alpha_{m}\right|\right)^{2}}\right)^{2}\right] .
$$

Application of the Cauchy-Buniakowsky-Schwarz inequality [38] to the right-hand side of the preceding equation results in Eq. (4.2b). The derivative of the optimum directivity satisfies

$$
\begin{aligned}
D^{\prime}(\alpha) & =\frac{\sqrt{P(\alpha)}}{T(\alpha)^{2}}\left[-2 T(\alpha)^{2}-\sqrt{P(\alpha)} T^{\prime}(\alpha)\right] \\
& =\sqrt{P(\alpha)} C^{\prime}(\alpha)
\end{aligned}
$$

which gives Eqs. (4.3a) and (4.5a). Differentiation of $D^{\prime}(\alpha) / C^{\prime}(\alpha)$ above with respect to $C(\alpha)$ yields Eq. (4.5b).

Equation (C7) implies that, for $\alpha \rightarrow \infty, D(\alpha)$ is either unbounded $(D \rightarrow \infty)$ or approaches a finite upper bound monotonically (as is seen to be true for the discrete case). For sketching an argument in favor of the former possibility, let $v(\alpha)=P(\alpha)^{-1 / 2}$. Equation (C4b) along with the definition of $D(\alpha)$ produce a trivial first-order differential equation for $v(\alpha)$, namely,

$$
v^{\prime}(\alpha)-D(\alpha)^{-1}=0, \quad v(0)=1,
$$

leading to

$$
\frac{1}{\sqrt{P(\alpha)}}=1+\int_{0}^{\alpha} \frac{d \xi}{D(\xi)}
$$

It is immediately inferred that if $\lim _{\alpha \rightarrow \infty} D(\alpha)=$ const, then $\alpha \sqrt{P(\alpha)}$ should be bounded for $\alpha \rightarrow \infty$. In view of the inequalities

$$
\frac{1}{2} \sum_{m=1}^{m_{0}}\left|\alpha_{m}\right|\left|A_{m}\right|^{2}<\alpha \sqrt{P(\alpha)}<\sum_{m=1}^{\infty}\left|\alpha_{m}\right|\left|A_{m}\right|^{2},
$$

where

$$
\begin{gathered}
\left|\alpha_{m}\right|>\alpha \text { for } m>m_{0}=m_{0}(\alpha), \\
\left|\alpha_{m}\right| \leqslant \alpha \text { otherwise, }
\end{gathered}
$$

$\alpha \sqrt{P}$ is bounded if and only if the series $\sum_{m=1}^{\infty}\left|\alpha_{m}\right|\left|A_{m}\right|^{2}$ converges. Let

$$
\psi_{m}(\hat{\mathbf{r}})=\frac{1}{V_{\mathcal{G}}} \int_{\mathcal{G}} d \mathbf{r}^{\prime} e^{-i k \hat{\mathbf{r}} \cdot \mathbf{r}^{\prime}} \mathcal{J}_{m}\left(\mathbf{r}^{\prime}\right), \quad m=1,2, \ldots
$$

Evidently, $\psi_{m}(\hat{\mathbf{r}})$ is smooth in $\hat{\mathbf{r}}$, expressing the far-field pattern of the "eigencurrent" $\mathcal{J}_{m}(\mathbf{r})$. The left-hand side of Eq. (B15) yields

$$
\frac{1}{\left|\alpha_{m}\right|}=\Omega_{0}^{-1} \int d \Omega \kappa(\hat{\mathbf{r}})\left|\psi_{m}(\hat{\mathbf{r}})\right|^{2}=\kappa\left(\hat{\mathbf{r}}_{m}\right)\left|\psi_{m}\left(\hat{\mathbf{r}}_{m}\right)\right|^{2},
$$

where $d \Omega=\sin \theta d \theta d \phi, \quad \kappa(\hat{\mathbf{r}})=\sin ^{2} \theta$, and $\Omega_{0}=4 \pi$ in three dimensions and $d \Omega=d \phi, \kappa(\hat{\mathbf{r}})=1$, and $\Omega_{0}=2 \pi$ in two dimensions. The introduction of the reasonable condition 


$$
\left|\psi_{m}\left(\hat{\mathbf{r}}_{m}\right)\right|^{2}=O\left(\left|\psi_{m}\left(\hat{\mathbf{r}}_{0}\right)\right|^{2}\right) \quad \text { for } \quad m \rightarrow \infty,
$$

at least for an infinite subsequence of $\{m=1,2, \ldots\}$, entails

$$
\frac{1}{\left|\alpha_{m}\right|} \leqslant O\left(\left|A_{m}\right|^{2}\right)
$$

The preceding condition is sufficient for the divergence of the series $\sum_{m=1}^{\infty}\left|\alpha_{m}\right|\left|A_{m}\right|^{2}$, contradicts the assumption of a bounded $\alpha \sqrt{P(\alpha)}$, and therefore ensures Eq. (4.3b). An investigation in mathematical rigor of the generality of Eq. (C14) or (C15) is beyond the scope of this paper.

\section{Discrete case}

Replacements (B34) and (B35) suggest that the limiting values of $C(\alpha)$ and $D(\alpha)$ for $\alpha \rightarrow \infty$ are finite. When $\alpha>-\left|\xi_{1}\right|$, Eqs. (C4)-(C7) and their direct consequences remain valid and Eqs. (B26)-(B29), once modified, readily furnish Eqs. (4.6)-(4.8). For $\alpha<-\left|\xi_{M}\right|$, the replacement

$$
\sqrt{P(\alpha)} \rightarrow-\sqrt{P(\alpha)}
$$

in Eqs. (C4), (C6), and (C7) yields Eqs. (4.9)-(4.11).

In order to derive Eq. (4.14), let $\alpha=-\epsilon-\left|\xi_{M}\right|$. For $\epsilon$ $\rightarrow 0^{+}$,

$\begin{aligned} P(\alpha)= & \left|X_{M}\right|^{4}\left|\xi_{M}\right|^{2} \epsilon^{-2}+2\left|X_{M}\right|^{2}\left|\xi_{M}\right| \sum_{m=1}^{M-1} \frac{\left|X_{m}\right|^{2}}{\left|\xi_{M}\right| /\left|\xi_{m}\right|-1} \epsilon^{-1} \\ & +O(1),\end{aligned}$

$$
N(\alpha)=\left|X_{M}\right|^{2}\left|\xi_{M}\right|^{2} \epsilon^{-2}+\sum_{m=1}^{M-1} \frac{\left|X_{m}\right|^{2}}{\left(\left|\xi_{M}\right| /\left|\xi_{m}\right|-1\right)^{2}}+O(\epsilon),
$$

$$
T(\alpha)=\left|X_{M}\right|^{2}\left|\xi_{M}\right| \epsilon^{-2}+\sum_{m=1}^{M-1} \frac{1}{\left|\xi_{m}\right|} \frac{\left|X_{m}\right|^{2}}{\left(\left|\xi_{M}\right| /\left|\xi_{m}\right|-1\right)^{2}}+O(\epsilon) .
$$

Equations (C18) and (C19) combined give

$$
\begin{aligned}
\epsilon= & \frac{\left|X_{M}\right|\left|\xi_{M}\right|}{\left[\sum_{m=1}^{M-1} \frac{\left|X_{m}\right|^{2}}{\left|\xi_{M}\right| /\left|\xi_{m}\right|-1}\right]^{1 / 2}} \sqrt{1-C /\left|\xi_{M}\right|} \\
& +O\left(\left(1-C /\left|\xi_{M}\right|\right)^{3 / 2}\right) .
\end{aligned}
$$

By use of Eq. (C17) and $\alpha=\alpha(C)$,

$$
\begin{aligned}
& D_{\text {opt }}(C)-D_{\text {opt }}\left(C_{\max }^{-}\right) \\
& =2\left|\xi_{M}\right|\left|X_{M}\right| \frac{\sum_{m=1}^{M-1} \frac{\left|X_{m}\right|^{2}}{\left|\sum_{M}\right| /\left|\xi_{m}\right|-1}}{\left[\sum_{m=1}^{M-1} \frac{\left|X_{m}\right|^{2}}{\left|\xi_{M}\right| /\left|\xi_{m}\right|-1}\right]^{1 / 2}} \sqrt{1-C /\left|\xi_{M}\right|} \\
& \quad+O\left(1-C /\left|\xi_{M}\right|\right) .
\end{aligned}
$$

In the first Riemann sheet, the positive square root is taken when $C$ lies in $\Delta_{C}=\left[\left|\xi_{1}\right|,\left|\xi_{M}\right|\right]$. Analytic continuation of $D_{\text {opt }}(C)$ to an adjacent sheet via crossing the branch cut that emanates from $C=\left|\xi_{M}\right|$ yields values $D_{\text {opt }}\left(C \in \Delta_{C}\right)$ below the optimum directivity. Therefore, the monotonically decreasing part of the optimum curve $D_{\text {opt }}(C)$, with a local maximum at $C=C_{\infty}$, is continued to a monotonically increasing curve below $D_{\text {opt }}\left(C_{\text {max }}\right)$.

It is of some interest to examine briefly the form of the optimum distribution $\mathbf{I}^{\text {(opt) }}$ when $k r_{j l}=k\left|\mathbf{r}_{j}-\mathbf{r}_{l}\right| \gg 1$ for all $j$ $\neq l\left(j, l=1,2, \ldots, M ; M\right.$ is fixed; $r \gg r_{j l}$ in the radiation zone). The off-diagonal elements of $\overline{\mathbf{K}}_{M}$ then become $O\left(\left(k r_{j l}\right)^{-1}\right)$. It is therefore concluded that for $m$ $=1,2, \ldots, M$,

$$
\left|\xi_{m}\right| \sim \begin{cases}3 M / 2 & \text { in three dimensions } \\ M & \text { in two dimensions. }\end{cases}
$$

The optimum distribution normalized to unity is

$$
\mathbf{I}^{(\mathrm{opt})} \sim \widetilde{\mathbf{I}}_{0},
$$

i.e., it approaches the uniform distribution. It follows that

$$
C(\alpha) \sim D(\alpha) \sim \begin{cases}3 M / 2 & \text { in three dimensions } \\ M & \text { in two dimensions. }\end{cases}
$$

These results corroborate the findings of Lo et al. [24], with their $Q$ being equal to $C / M$.

\section{APPENDIX D: AN ASYMPTOTIC EVALUATION OF $\varpi(k)$}

The integral

$$
\varpi(k)=\frac{1}{V_{\mathcal{G}}^{2}} \int_{\mathcal{G}} d \mathbf{r} \int_{\mathcal{G}} d \mathbf{r}^{\prime}\left|K\left(\mathbf{r}, \mathbf{r}^{\prime}\right)\right|^{2}
$$

gives a conservative estimate for the radius of convergence $R_{\alpha}=\left|\alpha_{1}\right|$ of the Neumann series (3.15) for $l \rightarrow \infty$. More precisely,

$$
R_{\alpha} \geqslant \varpi(k)^{-1 / 2} .
$$

More accurate estimates involve repeated integrals of $K\left(\mathbf{r}, \mathbf{r}^{\prime}\right)$ [31]. In this appendix, $\varpi(k)$ is studied systematically to leading order in $k^{-1}$ at high frequencies $(k \rightarrow \infty)$ by use of the Mellin transform technique [39]. Roughly speaking, "high frequencies" here means that the wavelength is much shorter than the maximum linear dimension of region $\mathcal{G}$. The analysis can be extended to higher orders in $k^{-1}$, but the calculations become more elaborate.

Let $h(t)$ be an integrable function defined on $0 \leqslant t<\infty$. The Mellin transform pair is given by

$$
\begin{gathered}
\bar{h}(\zeta)=\int_{0}^{\infty} d t t^{-\zeta} h(t), \\
h(t)=\frac{1}{2 \pi i} \int_{c-i \infty}^{c+i \infty} d \zeta t^{\zeta-1} \bar{h}(\zeta), \quad c \text { real, }
\end{gathered}
$$


where the integration path lies in the region of the $\zeta$ plane that ensures convergence of Eq. (D3). For the present purpose, consider the example

$$
h(t)= \begin{cases}t^{q-1}(\ln t)^{n} & \text { if } t \geqslant 1 \\ 0 & \text { if } 0 \leqslant t<1\end{cases}
$$

where $q$ is real and $n$ is an integer. Its Mellin transform is

$$
\bar{h}(\zeta)=\frac{\Gamma(n+1)}{(\zeta-q)^{n+1}}
$$

and $\operatorname{Re} \zeta>q$ is the region of integration for which Eq. (D3) makes sense. This example indicates that terms of the form $t^{q}(\ln t)^{n}$ in the asymptotic series of a complicated $h(t)$ for $t \rightarrow \infty$ can be found from the behavior of $\bar{h}(\zeta)$ near its poles lying to the left of the region for which Eq. (D4) is meaningful. This task, involving only power dependences in the vicinities of isolated points, is easier to carry out than dealing with logarithms in $t$ directly.

For the three-dimensional kernel, the Mellin transform of $\varpi(k)$ reads $[40]$

$$
\begin{aligned}
\overline{\boldsymbol{\varpi}}^{(3)}(\zeta)= & \frac{\pi}{4} 2^{-\zeta} \frac{\Gamma(1+\zeta) \Gamma\left(\frac{1-\zeta}{2}\right)}{\Gamma\left(1+\frac{\zeta}{2}\right)^{2} \Gamma\left(\frac{3+\zeta}{2}\right)} \frac{1}{V_{\mathcal{G}}^{2}} \int_{\mathcal{G}} d \mathbf{r} \int_{\mathcal{G}} d \mathbf{r}^{\prime}\left[1-(\hat{\mathbf{z}} \cdot \hat{\mathbf{R}})^{2}\right]^{2} R^{-1+\zeta} \\
& +\frac{\pi}{16} 2^{-\zeta} \frac{\Gamma(3+\zeta) \Gamma\left(\frac{1-\zeta}{2}\right)}{\Gamma\left(2+\frac{\zeta}{2}\right)^{2} \Gamma\left(\frac{7+\zeta}{2}\right)} \frac{1}{V_{\mathcal{G}}^{2}} \int_{\mathcal{G}} d \mathbf{r} \int_{\mathcal{G}} d \mathbf{r}^{\prime}\left[1-3(\hat{\mathbf{z}} \cdot \hat{\mathbf{R}})^{2}\right]^{2} R^{-1+\zeta} \\
& -\frac{\pi}{4} 2^{-\zeta} \frac{\Gamma(2+\zeta) \Gamma\left(\frac{1-\zeta}{2}\right)}{\Gamma\left(\frac{5+\zeta}{2}\right) \Gamma\left(2+\frac{\zeta}{2}\right) \Gamma\left(1+\frac{\zeta}{2}\right)} \frac{1}{V_{\mathcal{G}}^{2}} \int_{\mathcal{G}} d \mathbf{r} \int_{\mathcal{G}} d \mathbf{r}^{\prime}\left[1-(\hat{\mathbf{z}} \cdot \hat{\mathbf{R}})^{2}\right]\left[1-3(\hat{\mathbf{z}} \cdot \hat{\mathbf{R}})^{2}\right] R^{-1+\zeta}
\end{aligned}
$$

Note that the multiplicative factors of the $\Gamma$ functions have simple poles at all positive odd integers and at $\zeta=-1,-3$, -5 . For $k \rightarrow \infty$, only the negative poles are taken into account. Three cases need to be distinguished for the spatial integrals.

(i) If $\mathcal{G}$ is the interior of a closed surface, then all of the above integrals exhibit a pole at $\zeta=-2$. The inversion formula (D4) requires $-1<\operatorname{Re} \zeta<1$. In the neighborhood of the nearest left pole at $\zeta=-1$,

$$
\bar{\varpi}^{(3)}(\zeta) \sim \frac{1}{2} \frac{1}{\zeta+1} \frac{1}{V_{\mathcal{G}}^{2}} \int_{\mathcal{G}} d \mathbf{r} \int_{\mathcal{G}} d \mathbf{r}^{\prime} \frac{\left[1-(\hat{\mathbf{z}} \cdot \hat{\mathbf{R}})^{2}\right]^{2}}{R^{2}}
$$

By use of the pair (D5) and (D6), for $k \rightarrow \infty$,

$$
\boldsymbol{\varpi}^{(3)}(k) \sim \frac{1}{2} \frac{1}{V_{\mathcal{G}}^{2}} \int_{\mathcal{G}} d \mathbf{r} \int_{\mathcal{G}} d \mathbf{r}^{\prime} \frac{\left[1-(\hat{\mathbf{z}} \cdot \hat{\mathbf{R}})^{2}\right]^{2}}{(k R)^{2}} .
$$

It is noted in passing that this result may also be obtained via approximation of $K^{(3)}\left(\mathbf{r}, \mathbf{r}^{\prime}\right)$ in Eq. (D1) by its leading term $\left[1-(\hat{\mathbf{z}} \cdot \hat{\mathbf{R}})^{2}\right][\sin (k R) / k R]$ when $k R \gg 1$.

(ii) If $\mathcal{G}$ is a sufficiently smooth surface, all spatial integrals have a simple pole at $\zeta=-1$. In order to single this out, the inner integration is performed in the vicinity of $\mathbf{r}^{\prime}$ $=\mathbf{r}$ by use of a local tangent-normal system of axes, leading to

$$
\begin{aligned}
\overline{\boldsymbol{\varpi}}^{(3)}(\zeta) \sim & \frac{\pi}{8} \frac{1}{(\zeta+1)^{2}} \frac{1}{V_{\mathcal{G}}^{2}} \int_{\mathcal{G}} d \mathbf{r}\left[3+2(\hat{\mathbf{z}} \cdot \hat{\boldsymbol{\eta}})^{2}+3(\hat{\mathbf{z}} \cdot \hat{\boldsymbol{\eta}})^{4}\right] \\
& \text { for } \zeta \rightarrow-1,
\end{aligned}
$$

where $\hat{\boldsymbol{\eta}}=\hat{\boldsymbol{\eta}}(\mathbf{r})$ is a unit vector normal to $\mathcal{G}$ at point $\mathbf{r}$. By virtue of Eq. (D5), for $k \rightarrow \infty$,

$$
\boldsymbol{\varpi}^{(3)}(k) \sim \frac{\pi}{8} \frac{\ln k}{k^{2} V_{\mathcal{G}}}\left\{3+\frac{1}{V_{\mathcal{G}}} \int_{\mathcal{G}} d \mathbf{r}\left[2(\hat{\mathbf{z}} \cdot \hat{\boldsymbol{\eta}})^{2}+3(\hat{\mathbf{z}} \cdot \hat{\boldsymbol{\eta}})^{4}\right]\right\} .
$$

Note the appearance of the logarithm in $k$. When $\mathcal{G}$ lies entirely in the $x y$ plane, this leading term simplifies to

$$
\varpi^{(3)}(k) \sim \frac{\pi \ln k}{k^{2} V_{\mathcal{G}}} .
$$

(iii) If $\mathcal{G}$ is a curve $\mathbf{r}=\mathbf{r}(s)$ ( $s$ is the arc length, $0 \leqslant s$ $\leqslant V_{\mathcal{G}}$ ), all integrals exhibit a simple pole at $\zeta=0$. After some straightforward algebra,

$$
\varpi^{(3)}(k) \sim \frac{\pi}{15} \frac{1}{k V_{\mathcal{G}}}\left\{7-\frac{1}{V_{\mathcal{G}}} \int_{0}^{V_{\mathcal{G}}} d s\left[2(\hat{\mathbf{z}} \cdot \hat{\mathbf{u}})^{2}-3(\hat{\mathbf{z}} \cdot \hat{\mathbf{u}})^{4}\right]\right\},
$$


where $\hat{\mathbf{u}}=\hat{\mathbf{u}}(s)=|d \mathbf{r} / d s|^{-1}(d \mathbf{r} / d s)$ is a unit vector tangent to the curve at point $\mathbf{r}=\mathbf{r}(s)$. When $\mathcal{G}$ lies in the $x y$ plane, the leading term above reduces to

$$
\varpi^{(3)}(k) \sim \frac{7 \pi}{15} \frac{1}{k V_{\mathcal{G}}} .
$$

A similar analysis can be applied in two dimensions, where the Mellin transform of $\varpi(k)$ is [40]

$$
\overline{\boldsymbol{\varpi}}^{(2)}(\zeta)=2^{-\zeta} \frac{\Gamma(\zeta) \Gamma\left(\frac{1-\zeta}{2}\right)}{\Gamma\left(\frac{1+\zeta}{2}\right)^{3}} \frac{1}{V_{\mathcal{G}}^{2}} \int_{\mathcal{G}} d \mathbf{r} \int_{\mathcal{G}} d \mathbf{r}^{\prime} R^{-1+\zeta}
$$

The multiplicative factor of the $\Gamma$ functions contributes simple poles at all negative even integers, at $\zeta=0$, and at all positive odd integers. The inversion path is initially restricted in $0<\operatorname{Re} \zeta<1$. Two cases need to be considered.

(i) When $\mathcal{G}$ is the interior of a closed curve, the spatial integral is analytic at $\zeta=0$. Accordingly,

$$
\varpi^{(2)}(k) \sim \frac{1}{\pi} \frac{1}{V_{\mathcal{G}}^{2}} \int_{\mathcal{G}} d \mathbf{r} \int_{\mathcal{G}} d \mathbf{r}^{\prime} \frac{1}{k R} .
$$

In analogy with Eq. (D9), this result can be verified by replacing $K^{(2)}\left(\mathbf{r}, \mathbf{r}^{\prime}\right)=J_{0}(k R)$ in Eq. (D1) by the leading term $\sqrt{2 /(\pi k R)} \cos (k R-\pi / 4)$ when $k R \gg 1$.

(ii) If $\mathcal{G}$ is a curve, spatial integration reveals $\zeta=0$ as a double pole of $\overline{\boldsymbol{\varpi}}^{(2)}(\zeta)$, leading to

$$
\varpi^{(2)}(k) \sim \frac{2}{\pi} \frac{\ln k}{k V_{\mathcal{G}}} .
$$

\section{APPENDIX E: A FEW EXACTLY SOLUBLE CASES OF THE INTEGRAL EQUATION FOR THE OPTIMUM CURRENT}

In this appendix, the integral equation (3.4) is solved exactly in some simple cases where region $\mathcal{G}$ has a rotational symmetry. This is achieved by Fourier expanding in the associated angular variable $\phi$.

\section{Circular loop in two dimensions}

In two dimensions, consider a circle of radius $a$ centered at the origin. For definiteness, let $\hat{\mathbf{r}}_{0}$ be parallel to the positive $y$ axis, in the direction $\phi=\pi / 2$ of the associated polar coordinate system $(r, \phi)$. With $\widetilde{J}(\mathbf{r})=j(\phi)[j(\phi)$ periodic $]$, Eq. (3.4) reads

$$
j(\phi)+\frac{\alpha}{2 \pi} \int_{0}^{2 \pi} d \phi^{\prime} J_{0}\left(2 k a \sin \frac{\phi-\phi^{\prime}}{2}\right) j\left(\phi^{\prime}\right)=e^{i k a \sin \phi} .
$$

Substitution into this equation of

$$
j(\phi)=\sum_{n=-\infty}^{\infty} f_{n} e^{i n \phi}
$$

and use of the formulas

$$
e^{i k a \sin \phi}=\sum_{n=-\infty}^{\infty} J_{n}(k a) e^{i n \phi}
$$

$$
J_{0}\left(2 k a \sin \frac{\tau}{2}\right)=\sum_{m=-\infty}^{\infty} J_{m}(k a)^{2} e^{i m \tau}, \quad \tau=\phi-\phi^{\prime},
$$

yield decoupled equations for the coefficients $f_{n}$ :

$$
f_{n}=f_{n}(\alpha)=\frac{J_{n}(k a)}{1+\alpha J_{n}(k a)^{2}}, \quad n=-\infty, \ldots, \infty .
$$

The square of the optimum current norm is

$$
N=N(\alpha)=\sum_{n=-\infty}^{\infty}\left|f_{n}\right|^{2}=\sum_{n=-\infty}^{\infty} \frac{J_{n}(k a)^{2}}{\left[1+\alpha J_{n}(k a)^{2}\right]^{2}}
$$

The optimum scalar field equals

$$
\begin{aligned}
\psi(\hat{\mathbf{r}} ; \alpha) & =\frac{1}{2 \pi} \int_{0}^{2 \pi} d \phi^{\prime} j\left(\phi^{\prime} ; \alpha\right) e^{-i k a \cos \left(\phi-\phi^{\prime}\right)} \\
& =\sum_{n=-\infty}^{\infty} i^{-n} f_{n} J_{n}(k a) e^{i n \phi} \\
& =\sum_{n=-\infty}^{\infty} \frac{J_{n}(k a)^{2}}{1+\alpha J_{n}(k a)^{2}} e^{i n(\phi-\pi / 2)},
\end{aligned}
$$

where $\hat{\mathbf{r}}=(\cos \phi, \sin \phi)$. The total radiated power equals

$$
\begin{aligned}
T(\alpha) & =\frac{1}{2 \pi} \int_{0}^{2 \pi} d \phi|\psi(\hat{\mathbf{r}} ; \alpha)|^{2}=\sum_{n=-\infty}^{\infty}\left|f_{n}\right|^{2} J_{n}(k a)^{2} \\
& =\sum_{n=-\infty}^{\infty} \frac{J_{n}(k a)^{4}}{\left[1+\alpha J_{n}(k a)^{2}\right]^{2}} .
\end{aligned}
$$

Compare with Ref. [34].

\section{Circular disk in two dimensions}

With $\widetilde{J}(\mathbf{r})=j(r, \phi)(0 \leqslant r \leqslant a)$, the integral equation reads

$$
\begin{aligned}
& j(r, \phi)+\frac{\alpha}{\pi a^{2}} \int_{0}^{2 \pi} d \phi^{\prime} \int_{0}^{a} d r^{\prime} r^{\prime} \\
& \quad \times J_{0}\left(k \sqrt{r^{2}+r^{\prime 2}-2 r r^{\prime} \cos \left(\phi-\phi^{\prime}\right)}\right) j\left(r^{\prime}, \phi^{\prime}\right) \\
& =e^{i k r \sin \phi}
\end{aligned}
$$


Let

$$
j(r, \phi)=\sum_{n=-\infty}^{\infty} f_{n}(r) e^{i n \phi} .
$$

By invoking the addition formula

$$
J_{0}\left(k \sqrt{r^{2}+r^{\prime 2}-2 r r^{\prime} \cos \tau}\right)=\sum_{m=-\infty}^{\infty} J_{m}(k r) J_{m}\left(k r^{\prime}\right) e^{i m \tau}
$$

it is found by inspection that the $f_{n}(r)$ satisfy

$$
\begin{gathered}
f_{n}(r)=\left[1-\frac{2 \alpha}{a^{2}} \int_{0}^{a} d r^{\prime} r^{\prime} f_{n}\left(r^{\prime}\right) J_{n}\left(k r^{\prime}\right)\right] J_{n}(k r), \\
n=-\infty, \ldots, \infty
\end{gathered}
$$

This dependence on $r$ is expected because $j(r, \phi)$ should comply with Eq. (3.9). The direct substitution of $f_{n}(r)$ $=\lambda_{n} J_{n}(k r)$ into Eq. (E12) furnishes

$$
\lambda_{n}=\left[1+\frac{2 \alpha}{a^{2}} \int_{0}^{a} d r^{\prime} r^{\prime} J_{n}\left(k r^{\prime}\right)^{2}\right]^{-1}
$$

The remaining integration can be carried out explicitly by noticing that

$$
x J_{n}(x)^{2}=\frac{1}{2} \frac{d}{d x}\left\{x^{2}\left[J_{n}(x)^{2}-J_{n+1}(x) J_{n-1}(x)\right]\right\} .
$$

Finally,

$$
\begin{aligned}
j(r, \phi ; \alpha)= & \sum_{n=-\infty}^{\infty} \frac{J_{n}(k r)}{1+\alpha\left[J_{n}(k a)^{2}-J_{n+1}(k a) J_{n-1}(k a)\right]} \\
& \times e^{i n \phi},
\end{aligned}
$$

where $J_{n}(x)^{2}-J_{n+1}(x) J_{n-1}(x)>0$ for all real $x$. It follows that

$$
N(\alpha)=\sum_{n=-\infty}^{\infty} \frac{J_{n}(k a)^{2}-J_{n+1}(k a) J_{n-1}(k a)}{\left\{1+\alpha\left[J_{n}(k a)^{2}-J_{n+1}(k a) J_{n-1}(k a)\right]\right\}^{2}},
$$

$$
\begin{aligned}
\psi(\hat{\mathbf{r}} ; \alpha)= & \frac{1}{\pi a^{2}} \int_{0}^{2 \pi} d \phi^{\prime} \int_{0}^{a} d r^{\prime} r^{\prime} j\left(r^{\prime}, \phi^{\prime} ; \alpha\right) e^{-i k r^{\prime} \cos \left(\phi-\phi^{\prime}\right)} \\
= & \sum_{n=-\infty}^{\infty} \frac{J_{n}(k a)^{2}-J_{n+1}(k a) J_{n-1}(k a)}{1+\alpha\left[J_{n}(k a)^{2}-J_{n+1}(k a) J_{n-1}(k a)\right]} \\
& \times e^{i n(\phi-\pi / 2)}, \\
T(\alpha)= & \sum_{n=-\infty}^{\infty} \frac{\left[J_{n}(k a)^{2}-J_{n+1}(k a) J_{n-1}(k a)\right]^{2}}{\left\{1+\alpha\left[J_{n}(k a)^{2}-J_{n+1}(k a) J_{n-1}(k a)\right]\right\}^{2}} .
\end{aligned}
$$

Alternatively, by a comparison of Eq. (E11) to Eq. (B2), all of the above final formulas for the circle or disk can be easily reproduced through the formulation of Appendix B. For instance, it is recognized that the set of eigenvalues $\left\{\alpha_{m}\right\}_{m=1}^{\infty}$ for the disk is identified with $\left\{\bar{\alpha}_{n}\right\}_{n=0}^{\infty}$, where

$$
\bar{\alpha}_{n}=-\left[J_{n}(k a)^{2}-J_{n+1}(k a) J_{n-1}(k a)\right]^{-1}, \quad n=0,1, \ldots,
$$

each eigenvalue for $n \neq 0$ being doubly degenerate, with corresponding eigenfunctions $J_{n}(k r) e^{ \pm i n \phi}$. This set is not complete; an infinite set of linearly independent functions orthogonal to each eigenfunction can be constructed, for instance, from the basis $\left\{J_{n}\left(k_{n, p} r\right) e^{ \pm i n \phi} ; n\right.$ $=0,1, \ldots ; p=1,2, \ldots\}$, where $k_{l, 1} a, k_{l, 2} a, \ldots$ are the positive zeros of $J_{l}(x)$ arranged in ascending order of magnitude. The underlying reason for this lack of completeness is the description of the current by two independent space variables, as opposed to the far-field dependence on one space variable. Note that if $k$ coincides with $k_{l, p}$ for some $l$ and $p$, then $e^{i l \phi}$ becomes a nonradiating current for the circular loop of radius $a$. (See also Ref. [33].)

\section{Circular loop in three dimensions}

Consider a loop of radius $a$ centered at the origin. With the current polarization $(\hat{\mathbf{z}})$ chosen to be perpendicular to the plane of the loop and $\hat{\mathbf{r}}_{0}$ parallel to the positive $y$ axis, the integral equation (3.4) becomes

$$
\begin{gathered}
j(\phi)+\frac{\alpha}{2 \pi} \int_{0}^{2 \pi} d \phi^{\prime} \mathcal{K}\left(\phi-\phi^{\prime}\right) j\left(\phi^{\prime}\right)=e^{i k a \sin \phi} \\
\mathcal{K}(\tau)=\frac{\sin w}{w}+\frac{\cos w}{w^{2}}-\frac{\sin w}{w^{3}}=\frac{1}{4} \int_{-1}^{1} d \xi\left(1+\xi^{2}\right) e^{i w \xi}
\end{gathered}
$$

$$
w=w(\tau)=2 k a \sin \frac{\tau}{2}
$$

Starting with Eq. (E2) and following the previous steps lead to 


$$
\begin{aligned}
& f_{n}=\frac{J_{n}(k a)}{1+\alpha U_{n}(k a)}, \\
& U_{n}(k a)= \frac{1}{2 \pi} \int_{-\pi}^{\pi} d \tau \mathcal{K}(\tau) e^{-i n \tau}=\frac{1}{8 \pi} \int_{-1}^{1} d \xi\left(1+\xi^{2}\right) \int_{-\pi}^{\pi} d \tau e^{i w(\tau) \xi} \cos n \tau=\frac{1}{2} \int_{0}^{1} d \xi\left(1+\xi^{2}\right) J_{2 n}(2 k a \xi) \\
&= \frac{1}{4 k a}\left\{(2 n-1) \xi J_{2 n}(\xi) S_{-1,2 n-1}(\xi)-\xi J_{2 n-1}(\xi) S_{0,2 n}(\xi)+\frac{\xi}{(2 k a)^{2}}\left[(2 n+1) J_{2 n}(\xi)+(2 n+1)(2 n-1)^{2}\right.\right. \\
&\left.\left.\times J_{2 n}(\xi) S_{-1,2 n-1}(\xi)-\xi J_{2 n-1}(\xi)-\left(4 n^{2}-1\right) J_{2 n-1}(\xi) S_{0,2 n}(\xi)\right]\right\}\left.\right|_{\xi=0} ^{2 k a} \\
&= \frac{\pi}{4}\left[1+\frac{4 n^{2}-1}{(2 k a)^{2}}\right]\left\{J_{2 n-1}(2 k a)\left[\mathbf{E}_{2 n}(2 k a)+Y_{2 n}(2 k a)\right]-J_{2 n}(2 k a)\left[\mathbf{E}_{2 n-1}(2 k a)+Y_{2 n-1}(2 k a)\right]\right\} \\
&+\frac{1}{4 k a}\left[\frac{2 n+1}{2 k a} J_{2 n}(2 k a)-J_{2 n-1}(2 k a)+\frac{4 n^{2}-1}{(2 k a)^{2}}+1\right], \quad n=-\infty, \ldots, \infty,
\end{aligned}
$$

where $S_{\mu, \nu}$ is Lommel's function, $\mathbf{E}_{\nu}$ is Weber's function [40], and $U_{n}(k a)>0$ for all $k a$. With these $U_{n}(k a)$, it follows that

$$
\begin{gathered}
\psi(\hat{\mathbf{r}} ; \alpha)=\sum_{n=-\infty}^{\infty} \frac{J_{n}(k a) J_{n}(k a \sin \theta)}{1+\alpha U_{n}(k a)} e^{i n(\phi-\pi / 2)} \\
N(\alpha)=\sum_{n=-\infty}^{\infty} \frac{J_{n}(k a)^{2}}{\left[1+\alpha U_{n}(k a)\right]^{2}} \\
T(\alpha)=\sum_{n=-\infty}^{\infty} \frac{U_{n}(k a) J_{n}(k a)^{2}}{\left[1+\alpha U_{n}(k a)\right]^{2}}
\end{gathered}
$$

where $(r, \theta, \phi)$ is the usual spherical coordinate system. Again, compare with Eqs. (B2) and (B26)-(B28) of Appendix B.

Likewise, Eq. (3.19) can be solved explicitly in the case where a discrete rotational symmetry obtains, such as when filaments or dipoles are distributed uniformly along a circle, via application of the discrete Fourier transform. (In the engineering literature, this technique is known as the "method of symmetrical components"; see, for instance, Ref. [41].)

[1] C. W. Oseen, Ann. Phys. (Leipzig) 69, 202 (1922).

[2] A. Einstein, Phys. Z. 10, 817 (1909).

[3] A. Bloch, R. G. Medhurst, and S. D. Pool, Proc. IEE (London) 100, 303 (1953).

[4] L. La Paz and G. A. Miller, Proc. IRE 31, 214 (1943).

[5] C. J. Bouwkamp and N. G. de Bruijn, Philips Res. Rep. 1, 135 (1946).

[6] L. J. Chu, J. Appl. Phys. 19, 1163 (1948).

[7] R. F. Harrington, IRE Trans. Antennas Propag. AP-6, 219 (1958); R. F. Harrington, J. Res. Natl. Bur. Stand., Sect. D 64, 1 (1960); R. L. Fante, IEEE Trans. Antennas Propag. AP-17, 151 (1969); R. M. Kalafus, ibid. AP-17, 729 (1969); O. Einarsson, F. B. Sleator, and P. L. E. Uslenghi, ibid. AP-23, 28 (1975); R. L. Fante, ibid. AP-40, 1586 (1992).

[8] R. C. Hansen, Proc. IEEE 69, 170 (1981).

[9] J. S. McLean, IEEE Trans. Antennas Propag. AP-44, 672 (1996).

[10] D. R. Rhodes, IEEE Trans. Antennas Propag. AP-15, 568 (1967); G. V. Borgiotti, ibid. AP-15, 565 (1967); R. E. Collin, ibid. AP-15, 567 (1967); D. R. Rhodes, ibid. AP-20, 318 (1972); Synthesis of Planar Aperture Antennas (Oxford University Press, Oxford, 1974).
[11] D. R. Rhodes, J. Franklin Inst. 302, 225 (1976).

[12] R. E. Collin and S. Rothschild, Can. J. Phys. 41, 1967 (1963); J. M. Jarem, J. Math. Phys. 21, 2847 (1980); 23, 1713 (1982).

[13] R. E. Collin and S. Rothschild, IEEE Trans. Antennas Propag. AP-12, 23 (1964); G. Mitsioulis, Can. J. Phys. 69, 875 (1991).

[14] L. Solymár, Lectures on Electromagnetic Theory (Oxford University Press, Oxford, 1984).

[15] R. W. P. King, IEEE Trans. Antennas Propag. AP-37, 178 (1989).

[16] R. C. Hansen, IEEE Trans. Aerosp. Electron. Syst. 26, 345 (1990); Y. Huang, M. J. Lancaster, T. S. M. Maclean, Z. Wu, and N. McN. Alford, Physica C 180, 267 (1991); L. P. Ivrissimtzis, M. J. Lancaster, T. S. M. Maclean, and N. McN. Alford, IEEE Trans. Antennas Propag. AP-42, 1419 (1994).

[17] G. Fikioris, Ph.D. thesis, Harvard University, 1993 (unpublished); G. Fikioris, J. Electromagn. Waves Appl. 10, 307 (1996).

[18] V. Veremey and V. P. Shestopalov, Radio Sci. 26, 631 (1991).

[19] T. T. Taylor, IRE Trans. Antennas Propag. AP-3, 16 (1955).

[20] D. R. Rhodes, IEEE Trans. Antennas Propag. AP-11, 440 (1963).

[21] W. F. Richards and Y. T. Lo, IEEE Trans. Antennas Propag. AP-23, 165 (1975). 
[22] T. S. Angell, A. Kirsch, and R. E. Kleinman, Proc. IEEE 79, 1559 (1991).

[23] M. Uzsoky and L. Solymár, Acta Phys. Acad. Sci. Hung. 6, 185 (1956).

[24] Y. T. Lo, S. W. Lee, and Q. H. Lee, Proc. IEEE 54, 1033 (1966); 55, 292(E) (1967).

[25] T. S. Angell and R. E. Kleinman, J. Optim. Theory Appl. 37, 469 (1982); SIAM (Soc. Ind. Appl. Math.) J. Appl. Math. 44, 1246 (1984).

[26] A. Kirsch and P. Wilde, Math. Methods Appl. Sci. 10, 153 (1988); T. S. Angell and A. Kirsch, ibid. 15, 647 (1992); A. Jüschke, J. Jahn, and A. Kirsch, Comp. Optim. Appl. 7, 261 (1997).

[27] T. S. Angell, R. E. Kleinman, and B. Vainberg, SIAM (Soc. Ind. Appl. Math.) J. Appl. Math. (to be published); see also Ultra-Wideband, Short Pulse Electromagnetics 3, edited by C. E. Baum, L. Carin, and A. P. Stone (Plenum, New York, 1997), pp. 177-183.

[28] G. A. Deschamps and H. S. Cabayan, IEEE Trans. Antennas Propag. AP-20, 268 (1972).

[29] B. Z. Katsenelenbaum, Usp. Fiz. Nauk 164, 983 (1994) [Phys. Usp. 37, 903 (1994)].

[30] For fixed $k=2 \pi / \lambda$ and given maximum linear dimension $L_{\mathcal{G}}$ of region $\mathcal{G}$, it is of some interest to discuss briefly conditions under which Eq. (2.19), as well as Eqs. (2.9) and (2.10), surely provide good approximations for the field. The conditions $r$ $\gg \lambda, \quad r \gg L_{\mathcal{G}}$, and $r \gg L_{\mathcal{G}}^{2} / \lambda$, so that higher-order terms in the asymptotic expansion become negligible, are often considered adequate. However, in general, these tacitly assume a current moderately or slowly varying in $\mathcal{G}$, i.e., for instance, $|J|^{-1}\left|\nabla_{\mathcal{G}} J\right| \leqslant O(k)\left[\right.$ or $|J|^{-1}|\partial J / \partial s| \leqslant O(k)$ over a curve of arc length $s$ ]. For the purposes of this paper, the conditions should account for fields of currents reversing rapidly in space. For example, consider the two-dimensional distribution $J(\phi)$ $=e^{i n \phi}$ in a circle of radius $a$, where $\phi$ is the polar angle and $n$ is a positive integer. For polar distance $r>a$, the ensuing scalar field is $e^{i n \phi} J_{n}(k a) H_{n}^{(1)}(k r)$. The Hankel function can be replaced by its large-argument approximation only when $r$ $\gg n \lambda$. If $n$ is large, the latter condition guarantees a transition to the radiation zone.

[31] F. G. Tricomi, Integral Equations (Dover, New York, 1985).

[32] J. B. Arnett and G. H. Goedecke, Phys. Rev. 168, 1424 (1968); A. J. Devaney and E. Wolf, Phys. Rev. D 8, 1044 (1973); N. Bleistein and J. K. Cohen, J. Math. Phys. 18, 194 (1977).

[33] B. Z. Katsenelenbaum, Radiotekh. Elektron. 38, 998 (1993) [J. Commun. Technol. Electron. 38, 112 (1993)].

[34] B. Z. Katsenelenbaum and M. Yu. Shalukhin, Radiotekh. Elektron. 33, 1878 (1988) [ Sov. J. Commun. Technol. Electron. 34, 25 (1989)].

[35] A. I. Uzkov C. R. (Dokl.) Acad. Sci. URSS 53, 35 (1946).

[36] G. Fikioris, R. W. P. King, and T. T. Wu, J. Appl. Phys. 68, 431 (1990).

[37] C. Müller, Foundations of the Mathematical Theory of Electromagnetic Waves (Springer-Verlag, Berlin, 1969), pp. 8687.

[38] N. I. Akhiezer and I. M. Glazman, Theory of Linear Operators in Hilbert Space, Vol. I (Dover, New York, 1993), p. 2.

[39] J. D. Bjorken and T. T. Wu, Phys. Rev. 130, 2566 (1963).

[40] Bateman Manuscript Project, Higher Transcendental Functions, Vol. II, edited by A. Erdélyi (Krieger, Malabar, FL, 1981), pp. 51, 35-37, 40-42, and 90.

[41] R. W. P. King, R. B. Mack, and S. S. Sandler, Arrays of Cylindrical Dipoles (Cambridge University Press, London, 1968), Chap. 4. 